\title{
Quality of Peach (Prunus persica L.) Genotypes Packed in LDPE Plastic Packaging under Different Storage Conditions
}

\section{Tajebe Mosie (1æ $^{\rtimes}$ \\ Kebede Woldetsadik ${ }^{2}$ Mulualem Azene ${ }^{3}$}

'Holeta Agricultural Research Center; Temperate and Indigenous Fruits Research Program, Holeta, Ethiopia. Email:tajebemu@gmail.com Tel:+251920503991

${ }^{\circ}$ Haramaya University, College of Agriculture and Environmental Science, School of Plant Sciences, Dire Dawa, Ethiopia.

Email:kwolde58@gmail.com Tel:+251915321047

Ambo University, College of Agriculture and Veterinary Science, Department of Horticulture, Ambo, Ethiopia.

Email:mulualem2011@gmail.com Tel:+251946379198

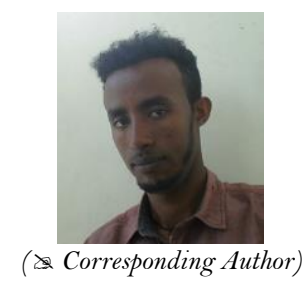

( Corresponding Author)

\begin{abstract}
Peach Marketing in Ethiopia is complicated by postharvest losses like other horticulture produce. Although peach varieties have been introduced and studied for their productivity, their postharvest quality has not been evaluated at Holeta. Therefore, the experiment was done to assess influence of polyethylene plastic packaging and storage conditions on postharvest quality and storage life stability of peach genotypes. It was conducted at Holeta agricultural research center from January to February, 2018. Peach genotypes 'Bonnigold, Early Grande, 90-19H and Topic Beauty' packed in perforated and non-perforated low density polyethylene plastic (LDPE) and stored under two storage conditions i.e. ambient $\left(6.4^{\circ} \mathrm{C}\right.$ to $\left.20.2{ }^{\circ} \mathrm{C} ; 40 \%-71 \% \mathrm{RH}\right)$ and cold storage $\left(-1{ }^{\circ} \mathrm{C}\right.$ to $\left.1{ }^{\circ} \mathrm{C} ; 85-99 \% \mathrm{RH}\right)$. Genotypes had different response to packaging materials and storage conditions. Perforated bag packaging with cold storage was found more effective as compared to non-perforated and the control fruits in maintaining firmness. Non-perforated bag packaging's combined with cold storage extended shelf life of peach fruits $10-20$ days compared to other treatment combinations. The non-perforated plastic packaged fruits stored in the cold storage also maintained more TSS, AA, TA, TSS/TA, pH, marketability and specific gravity content and lowered weight loss and decay. Therefore, non-perforated plastic packaging with cold storage condition can be considered in maintaining its quality and extending the shelf life of peaches mainly for commercial producers, traders as well as research stations.
\end{abstract}

Keywords: Packaging films, Peach, Quality, Storage condition, Genotypes, Perforated plastic, Shelf life.

Citation | Tajebe Mosie; Kebede Woldetsadik; Mulualem Azene (2019). Quality of Peach (Prunus persica L.) Genotypes Packed in LDPE Plastic Packaging under Different Storage Conditions. Agriculture and Food Sciences Research, 6(2): 182-197.

History:

Received: 5 April 2019

Revised: 15 May 2019

Accepted: 25 June 2019

Published: 20 August 2019

Licensed: This work is licensed under a Creative Commons Attribution 3.0 License $(\mathrm{cc})$ EY

Publisher: Asian Online Journal Publishing Group
Acknowledgement: The authors express their heartfelt gratitude for Holeta agricultural research center staff members particularly horticulture case team for their valuable contribution for the accomplishment of this research.

Funding: This study is funded by Ethiopian Institute of Agricultural Research.

Competing Interests: The authors declare that they have no conflict of Competing

Transparency: The authors confirm that the manuscript is an honest, accurate, and transparent account of the study was reported; that no vital features of the study have been omitted; and that any discrepancies from the study as planned have been explained.

Ethical: This study follows all ethical practices during writing.

\section{Contents}

1. Introduction

2. Materials and Methods...

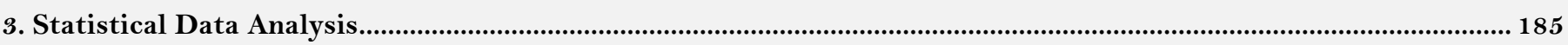

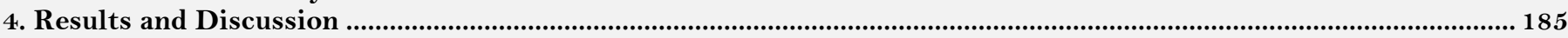

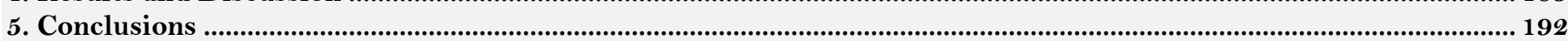

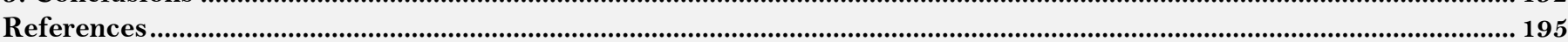




\section{Contribution of this paper to the literature}

This study contributes to literature by evaluating the quality and shelf life stability of peach (prunus persica) genotypes packed in LDPE plastic packaging.

\section{Introduction}

Peach (prunus persica (L.) Batsch) is mainly grown in temperate regions and is the most important among the stone fruits [1]. It is a versatile fruit with good source of antioxidants Byrne [2] and Cantin, et al. [3]. Peaches consumption has increased due to high sources of sugars, organic acids, minerals and vitamins.

Ethiopian highlands are endowed with a mosaic of soils and climate which are suitable for the production of many temperate fruits and nut crops. The country is characterized by having diverse topography and agroecological zones, of which over $50 \%$ of the total area is highland with elevation between $2000-4500$ masl with adequate water resources and low temperature during winter that can favor many temperate fruit crops to grow [4]. Ethiopia's share of peach and nectarine production in the world is negligible (172 tons and area coverage of 31 hectare). However, equations show increasing in area as well as production compared to previous years [5].

Peach is a climacteric fruit that have short shelf life under room temperature due to their high respiratory rate and fast ripening process. The ripening of peaches is complex that cannot be measured by a single factor. Moreover, its marketing in Ethiopia is complicated by high postharvest losses like other fresh produces. A postharvest loss of about 30\% is reported for fresh produce in Ethiopia [6]. This postharvest loss is due to poor storage facilities coupled with improper packaging and transportation [7].

Peaches are highly perishable that do not able to prolonged storage. Generally, peach varieties have a very short storage potential due to fast ripening process; which results in a limited time for commercialization. High temperature combined with low relative humidity during harvesting and marketing are the major factors which reduce the post-harvest life of peaches. Under ambient storage conditions the life of peach does not exceed 3-5 days $[8]$.

Refrigerator storage is chosen for extending the commercial life of peach fruits [9]. Sound, well matured peach fruits can be stored for $14-42$ days under $-0.6-\mathrm{O}^{\circ} \mathrm{C}$ temperature range [10]. On the other hand, if stored too long at or near $\mathrm{O}{ }^{\circ} \mathrm{C}$, they are subjected to chilling injury. The start of such symptoms determines the postharvest storage capacity since chilling injury can reduce consumer acceptance [11]. Storage at low temperatures, mainly with the range of $2.2-5^{\circ} \mathrm{C}$, is prone to physiological disorder known as internal breakdown [12] which is a symptom associated with chilling injury [12]. Many activities have been studied to reduce the incidence of physiological disorder in peach cultivars [12] including cold room with controlled atmosphere (CA) storage. CA storage has shown the reduction of mealiness in peach and nectarine cultivars, mainly with high $\mathrm{CO}_{2}$ and reduced $\mathrm{O}_{2}$ levels [13].

Packages during harvesting and postharvest handling of fresh produce are used to reduce damage and postharvest loss [14]. Packing of fruits in polymeric films creates modified atmospheric conditions (MAP) inside the package which results in reduced rate of respiration, transpiration, delaying ripening and other metabolic processes of fruits [15]. This packaging technique is accepted now as the technology of future. Changes that result in loss in quality occur during postharvest storage of fresh produce as a result of internal and external factors. Therefore, use of environmentally friendly technologies such as cold storage, waxing, calcium dipping, packaging and use of plant extracts can be used to delay the adverse effects [16].

Postharvest factors responsible for development of browning are the picking date, the duration of the cooling period, the $\mathrm{CO}_{2}$ and $\mathrm{O}_{2}$ partial pressure, the storage temperature and condition [17]. Although peach varieties have been introduced and evaluated for their productivity, their postharvest quality was not evaluated in our country. Besides, efforts have not been done to evaluate the influence of postharvest handling practices to maintain peach fruit quality and shelf life at Holeta agricultural research center; local producers store the fruits in pure water till transported to market. Thus the objective of this study was to evaluate the quality of peach genotypes packed in plastic packaging under different storage conditions at Holeta, Central Ethiopia.

\section{Materials and Methods \\ 2.1. Experimental Sites}

This study was conducted at Holeta Agricultural Research Center (HARC) which is situated at an altitude of 2400 masl $09^{`} \mathrm{~N}$ latitude and $38^{`} 29 \mathrm{E}$ longitude. The area is characterized by mean annual rainfall of $1041.4 \mathrm{~mm}$ and relative humidity of $58.7 \%$. The main rainy season is June to September, which accounts for $70 \%$ of the rainfall while the remaining $30 \%$ is from February to April. The average annual maximum and minimum temperature is $21.7^{\circ} \mathrm{C}$ and $6.7{ }^{\circ} \mathrm{C}$, respectively [18].

\subsection{Treatments and Experimental Design}

The treatments consisted of 4x3x2 factorial combinations of peach genotypes (Tropic Beauty, 90-19H, Bonnigold and Early Grande), LDPE plastic packaging (perforated plastic, non-perforated plastic and nonpackaged) and storage conditions (cold and ambient). Completely randomized design (CRD) with factorial arrangement with three replications was used. The experiment was conducted between January and February 2018 at Holeta Agricultural Research center horticulture laboratory.

\subsection{Experimental Procedure}

Peach fruits were obtained from orchard of Holeta Agricultural Research Center. Fruits were harvested from 13 years old trees that received all the necessary management practices (pruning, training, fertilization and irrigation) uniformly. Fruits were harvested when surface color changed from green to yellow and shape with fullness of shoulder and suture [19]. 


\subsection{Data Collection}

Three fruits of sample per treatment were taken to laboratory for chemical analysis at five days interval. Ten fruits were kept for non-destructive evaluation per treatment.

\subsubsection{Physiological Loss in Weight (PLW)}

Physiological loss in weight was determined using the methods described by Mohammed, et al. [20]. Weight loss was calculated using the formula:

$$
\text { weight loss }(\%)=\frac{\text { initial weight-final weight }}{\text { initial weight }} \times 100
$$

\subsubsection{Total Soluble Solid}

It was determined in the laboratory by extracting the juice of three peach fruits flesh using juice extractor. The reading of TSS was taken from hand held refract- meter with a reading of 0-32 ${ }^{\circ}$ Brix.

\subsection{3. pHand Titratable Acidity (TA)}

The $\mathrm{pH}$ was measured with $\mathrm{pH}$ meter and TA was obtained by using the method of Garner, et al. [21]. The acidity was calculated with the following formula:

$$
\% \text { acidity }=\frac{[\text { mls of } \mathrm{NaOH}] \times[0.1 \mathrm{~N} \mathrm{NaOH}][\text { milliequivalent factor }][100]}{\text { grams of sample }}
$$

Where, a stone fruit predominant acid is malic acid and its milli equivalent factor is 0.067.

\subsubsection{TSS/Acid Ratio (Ripening Index):}

It was determined by dividing total soluble solid taken from refractometer reading ( ${ }^{\circ}$ Brix) by percent acidity obtained in titratable acidity.

\subsubsection{Ascorbic Acid (AA)}

It was measured by using a modified method of Bessey and King [22]. Concentration of vitamin $\mathrm{C}$ in peach flesh juice was determined by the following equation:

Conc. of vitamin $\mathrm{C}$ in juice $\left(\frac{\mathrm{mg}}{\mathrm{ml}}\right)=\frac{D C P I P(\mathrm{ml}) \text { used to titrate peach juice }}{D C P I P(\mathrm{ml}) \text { used to titrate standard }} \times 1 \mathrm{mg} / \mathrm{ml}$

Ascorbic acid content was expressed on a fresh weight basis as $m g 100 \mathrm{~g}^{-1}$ of juice.

\subsubsection{Firmness}

Firmness of peach fruit was assessed using the subjective method by pressing the fruit with the thumb and forefinger [23]. The firmness of fruit was measured based on the resistance of finger pressure and scored by partially trained panelists, using a 1 to 5 scale which represents five firmness classes. The firmness classes was represented as $1=$ extra soft, $2=$ soft, $3=$ firm, $4=$ hard, and $5=$ extra hard.

\subsubsection{Fruit Marketability}

Was assessed subjectively using the procedure described by Mohammed, et al. [20]. The descriptive quality attributes were determined by observing the level of decay, color, surface defects and shriveling. A 1-9 ratings, with $1=$ unusable, $3=$ unmarketable, $5=$ fair, $7=\operatorname{good}$ and $9=$ excellent, was used to evaluate the fruit quality. Fruits receiving a rating of five and above were considered as marketable, while those rated less than five were unmarketable. The number of marketable fruits was used as a measure to calculate the percentage of marketable fruits during storage.

$$
\text { Percentage marketability }=\frac{\text { number of marketable fruits }}{\text { total number of fruits }} \times 100
$$

\subsubsection{Percentage Decay}

Percentage decay is the number of decayed fruits due to micro-organisms infection which was recorded and calculated as a percentage of the total number of fruits using the following equation. Fruit was considered as rotten when more than $5 \%$ of surface area was decayed, blackened or rotten [24].

$$
\text { Percentage decay }=\frac{\text { number of decayed fruit }}{\text { total number of fruit }} \times 100
$$

\subsubsection{Specific Gravity ( $S G$ )}

Was determined by converting total soluble solid reading expressed with ${ }^{\circ}$ Brix using the following simple equation [25]:

$$
\mathrm{SG}=1+\left(0.004 \mathrm{x}{ }^{\circ} \text { Brix }\right)
$$

Where, ${ }^{\circ}$ Brix $=$ TSS reading, $\mathrm{SG}=$ specific gravity and 0.004 gravity point (constant).

\subsubsection{Flesh Color Browning}

Fruits were visually evaluated for flesh color browning and scored by sensory panelists, using a 1-6 scale which presents six flesh browning stages of peach. The six browning stages was represented as $1=$ none, $2=$ very slight browning in the pit cavity, $3=$ slight browning in the pit cavity and surrounding tissue, $4=$ moderate browning on less than $50 \%$ of the flesh, $5=$ severe browning on $50-75 \%$ of the flesh, and $6=$ extreme browning covering most of the flesh [26]. 


\section{Statistical Data Analysis}

Analysis of variance (ANOVA) for the parameters was done using SAS statistical software and comparison of treatment means was made by Least Significance difference Test at $5 \%$ probability level.

\section{Results and Discussion}

\subsection{The Storage Conditions}

Temperature $\left(\mathrm{T}^{\circ}\right)$ and relative humidity $(\mathrm{RH})$ of both ambient and cold storage conditions were recorded during the storage period. Under ambient condition temperature were varied between $6.4^{\circ} \mathrm{C}$ and $20.2{ }^{\circ} \mathrm{C}$ with the average of $13.3^{\circ} \mathrm{C}$ while the cold storage temperature was maintained between -1 and $1{ }^{\circ} \mathrm{C}$. The relative humidity of the storage environment ranged between $40 \%$ and $71 \%$ under the ambient conditions, with average $55.5 \%$ while it ranged between $85 \%$ and $99 \%$ in cold store with the average being $92 \%$.

\subsection{Physiological Loss in Weight}

There was highly significant $(\mathrm{P} \leq 0.001)$ difference in physiological loss in weight (PLW) due to the combined effect of packaging and storage condition on peach fruit genotypes throughout the storage period Table 1 . The weight loss values varied between 0.01 to $6.3 \%$ on day 5 and from $0.30 \%$ to total loss on day 10 .

Generally, Weight loss of peach fruits was increased during the storage period both under cold and ambient conditions. On day 5, weight loss of non-packaged fruits of Early Grande variety stored at ambient condition was $6.30 \%$ which was significantly $(\mathrm{P} \leq 0.001)$ higher than weight loss of peach fruits subjected to all other treatments on the same date. Fruits packaged with perforated plastic and stored at ambient conditions had a relatively higher weight loss next to non-packaged ones. The highest weight loss was recorded from the control fruits of Early Grande variety stored at cold storage, whereas the lowest was for fruits of 90-19H without packaging and stored in the cold storage on day 10. From day 10 onwards, fruits of all cultivars kept ambient condition without packaging were unmarketable whereas all cultivars kept in cold store were in good condition up to the last storage period (30 days) with PWL ranging from 0.3\% (Tropic Beauty packaged in perforated plastic) to $3.37 \%$ in cultivar $90-19 \mathrm{H}$ packaged in perforated plastic. Non-perforated plastic in cold store kept fruit PWL less than $1 \%$ while in the perforated plastic combined with cold store it reached $2.14 \%$ (Tropic Beauty) to $3.37 \%$ (90-19H) on day 30 of storage. In general, peach fruits packed with non-perforated plastic bags showed lower weight loss both under ambient and cold storage compared to perforated plastic and control in respective storage conditions. This could be due to less availability of $\mathrm{O}_{2}$ inside non-perforated packed fruits for respiration. Therefore, reduction in the rate of respiration which ultimately caused decrease and increase in $\mathrm{O}_{2}$ and $\mathrm{CO}_{2}$ levels, respectively, as storage time advances. The balanced levels of $\mathrm{CO}_{2}$ and $\mathrm{O}_{2}$ inside the package after initial storage could cause marked changes in the activities of specific enzymes [27]. After day 5, nearly all non-packed fruits stored under ambient conditions were unmarketable while those fruits in cold storage kept well for over 10 days.

Generally, weight loss of fruits was higher under ambient condition than in the cold storage which is varied with genotypes. Lower weight loss in non-perforated polyethylene packed fruits might be due to less availability of oxygen for respiration. The present results are in agreement with the findings of Calvo, et al. [28] and Tijskens and Vollebregt [29]. Weight loss differences among the treatments also appear to be due to differences in temperature and relative humidity among the storage conditions. Hence reduced rate of respiration and transpiration at lower temperature and higher relative humidity $(\mathrm{RH})$ could be the reason for such reduced rate of weight loss of fruits in the cold storage [30]. About 10.0\% physiological loss in weight is considered as an index of termination of shelf life of commodities [31].

High storage temperature leads to accelerated water loss and subsequently to shriveling and softening of the fruit [32]. High temperature contributed to high water loss of peach in ambient condition which is associated with faster metabolism; increased cell wall degradation and higher membrane permeability leading to easy evaporation [33].

Furthermore, lower weight loss of fruits in the package could be due to slow rate of ripening and prevention of excessive moisture loss. Similar results were also presented by Tilahunsiyum and Kebede [34] and Nath, et al. [35]. Relatively lower water vapor transmission rate of non-perforated plastic may also contribute for the development of relative higher humidity inside the package Mathooko [36]. According to Ben-Yehoshua [37] the main function of packaging is to reduce respiration rate and water loss by transpiration which could affect the fruits metabolism. This might have led to the extension of shelf life of peach fruits in non-perforated plastic as compared to control and perforated packed fruits. The present findings are in agreement with the previous findings of Ribeiro, et al. [38] and Drake, et al. [39].

\subsection{Total Soluble Solids}

The values in total soluble solids (TSS) of peach fruits during the storage periods are displayed in Table 2. There was significant $(\mathrm{P} \leq 0.05)$ interaction effect of storage condition, genotype and packaging on the TSS values of fruits which varied between $8.3-13.33{ }^{\circ}$ Brix in the cold storage and from 8.33-12.4 ${ }^{\circ}$ Brix under ambient conditions. There is high consumer acceptance of peaches with high soluble solids; for mid-season peach cultivars a minimum of $11.0 \%$ TSS is considered optimum [40]. TSS value showed increment with storage duration in respective treatments. Acceptable TSS values were attained in Bonnigold and Early Grande on day five while the remaining cultivars required 10 to 15 days to reach similar levels. In general, non-packaged fruits had relatively higher TSS while lower values were recorded in non-perforated plastic packed fruits. In cold storage, Bonnigold variety with perforated plastic packs had the highest TSS content (13.33) while least value was recorded in 90-19H (11.07) at the end of storage. Increase in TSS during storage might be associated with starch hydrolysis of fruits $[41]$. 
Table-1. Influence of plastic packaging and storage condition on PLW of peach genotypes during the storage period.

\begin{tabular}{|c|c|c|c|c|c|c|c|c|c|}
\hline \multicolumn{3}{|c|}{ Treatments } & & \multicolumn{6}{|c|}{ Storage period(days) } \\
\hline Genotypes & $\begin{array}{c}\text { Plastic } \\
\text { Packaging }\end{array}$ & Storage & $\mathrm{O}$ & 5 & 10 & 15 & 20 & 25 & 30 \\
\hline \multirow[t]{6}{*}{ Tropic Beauty } & \multirow[t]{2}{*}{ Perforated } & Cold & 0.00 & $0.44^{\mathrm{ij}}$ & $1.01^{\mathrm{i}}$ & $1.17^{\mathrm{h}}$ & $1.83^{\mathrm{d}}$ & $2.07^{\mathrm{d}}$ & $2.14^{\mathrm{d}}$ \\
\hline & & Ambient & 0.00 & $0.53^{\mathrm{i}}$ & $1.23^{\mathrm{h}}$ & $1.78^{\mathrm{f}}$ & - & - & - \\
\hline & \multirow{2}{*}{$\begin{array}{c}\text { Non- } \\
\text { perforated }\end{array}$} & Cold & 0.00 & $0.01^{\mathrm{m}}$ & $0.30^{\mathrm{m}}$ & $0.31^{1}$ & $0.5 \mathrm{O}^{\mathrm{i}}$ & $0.59^{h}$ & $0.62^{\mathrm{h}}$ \\
\hline & & Ambient & 0.00 & $0.17^{\mathrm{klm}}$ & $0.41^{1}$ & $0.47^{k}$ & $0.72^{\mathrm{g}}$ & - & - \\
\hline & \multirow{2}{*}{$\begin{array}{c}\text { Non- } \\
\text { packaged }\end{array}$} & Cold & 0.00 & $2.38^{\mathrm{e}}$ & $10.54^{b}$ & - & - & - & - \\
\hline & & Ambient & 0.00 & $4.08^{\mathrm{c}}$ & - & - & - & - & - \\
\hline \multirow[t]{6}{*}{$9 \mathrm{O}-19 \mathrm{H}$} & \multirow[t]{2}{*}{ Perforated } & Cold & 0.00 & $0.29^{\mathrm{jk}}$ & $2.46^{\mathrm{e}}$ & $2.60^{\mathrm{c}}$ & $3.29^{\mathrm{a}}$ & $3.35^{\mathrm{a}}$ & $3.37^{\mathrm{a}}$ \\
\hline & & Ambient & 0.00 & $0.77^{\mathrm{h}}$ & $3.47^{\mathrm{d}}$ & $3.95^{\mathrm{a}}$ & - & - & - \\
\hline & \multirow{2}{*}{$\begin{array}{c}\text { Non- } \\
\text { perforated }\end{array}$} & Cold & 0.00 & $0.02^{\operatorname{lm}}$ & $0.60^{\mathrm{k}}$ & $0.68^{j}$ & $0.74 \mathrm{~g}$ & $0.78^{f}$ & $0.80^{f}$ \\
\hline & & Ambient & 0.00 & $0.12^{\mathrm{klm}}$ & $0.81^{\mathrm{j}}$ & $1.25^{\mathrm{h}}$ & $1.44^{\mathrm{e}}$ & - & - \\
\hline & \multirow{2}{*}{$\begin{array}{c}\text { Non- } \\
\text { packaged }\end{array}$} & Cold & 0.00 & $3.53^{\mathrm{d}}$ & $10.35^{\mathrm{c}}$ & - & - & - & - \\
\hline & & Ambient & 0.00 & $5.66^{\mathrm{b}}$ & - & - & - & - & - \\
\hline \multirow[t]{6}{*}{ Bonnigold } & \multirow[t]{2}{*}{ Perforated } & Cold & 0.00 & $0.81^{\mathrm{h}}$ & $1.19^{\mathrm{h}}$ & $2.53^{\mathrm{c}}$ & $2.90^{\mathrm{b}}$ & $2.94^{\mathrm{b}}$ & $2.96^{\mathrm{b}}$ \\
\hline & & Ambient & 0.00 & $0.12^{\mathrm{klm}}$ & $1.83^{\mathrm{f}}$ & $2.27^{\mathrm{d}}$ & - & - & - \\
\hline & \multirow{2}{*}{$\begin{array}{c}\text { Non- } \\
\text { perforated }\end{array}$} & Cold & 0.00 & $0.01^{\mathrm{m}}$ & $0.49^{1}$ & $0.55^{\mathrm{k}}$ & $0.90^{f}$ & $0.94^{\mathrm{e}}$ & $0.96^{\mathrm{e}}$ \\
\hline & & Ambient & 0.00 & $0.13^{\mathrm{klm}}$ & $0.85^{j}$ & $1.63^{\mathrm{g}}$ & $1.82^{\mathrm{d}}$ & - & - \\
\hline & \multirow{2}{*}{$\begin{array}{c}\text { Non- } \\
\text { packaged }\end{array}$} & Cold & 0.00 & $1.29^{\mathrm{g}}$ & $10.42^{\mathrm{c}}$ & - & - & - & - \\
\hline & & Ambient & 0.00 & $5.60^{\mathrm{b}}$ & - & - & - & - & - \\
\hline \multirow[t]{6}{*}{ Early Grande } & \multirow[t]{2}{*}{ Perforated } & Cold & 0.00 & $0.23 \mathrm{jkl}$ & $1.58^{\mathrm{g}}$ & $2.00^{\mathrm{e}}$ & $2.11^{\mathrm{c}}$ & $2.18^{\mathrm{c}}$ & $2.20^{\mathrm{c}}$ \\
\hline & & Ambient & 0.00 & $0.60^{h i}$ & $2.48^{\mathrm{e}}$ & $2.98^{\mathrm{b}}$ & - & - & - \\
\hline & \multirow{2}{*}{$\begin{array}{c}\text { Non- } \\
\text { perforated }\end{array}$} & Cold & 0.00 & $0.02^{\operatorname{lm}}$ & $0.47^{1}$ & $0.56^{\mathrm{k}}$ & $0.59^{\mathrm{h}}$ & $0.67^{g}$ & $0.69^{g}$ \\
\hline & & Ambient & 0.00 & $0.19^{\mathrm{klm}}$ & $0.84^{\mathrm{j}}$ & $0.91^{\mathrm{i}}$ & $0.95^{\mathrm{f}}$ & - & - \\
\hline & \multirow{2}{*}{$\begin{array}{c}\text { Non- } \\
\text { packaged }\end{array}$} & Cold & 0.00 & $1.63^{\mathrm{f}}$ & $10.95^{\mathrm{a}}$ & - & - & - & - \\
\hline & & Ambient & 0.00 & $6.30^{\mathrm{a}}$ & - & - & - & - & - \\
\hline \multicolumn{3}{|c|}{$\mathrm{SE} \pm$} & - & 0.08 & 0.03 & 0.03 & 0.02 & 0.02 & 0.02 \\
\hline \multicolumn{3}{|c|}{ CV (\%) } & - & 9.10 & 1.88 & 3.54 & 2.57 & 1.97 & 1.53 \\
\hline
\end{tabular}

On day 5 the control fruits of Bonnigold stored at ambient conditions had the highest TSS value (11.33 $\left.{ }^{\circ} \mathrm{Brix}\right)$ while Tropic Beauty had the lowest TSS value (9.27). Likewise, on day 10, control fruits of Bonnigold stored in the cold storage attained the highest TSS content (13.33 ${ }^{\circ}$ Brix) while Tropic Beauty under similar condition had the lowest TSS content (9.53). This could be due to accelerated ripening because of higher temperature at ambient conditions and free access of the non-packed fruits to $\mathrm{O}_{2}$ which increase respiration rates, resulting in faster conversion of starch to soluble sugars [30]. The variations of values among the cultivars show differences in their genetic makeup and response to storage conditions.

Perforated LDPE bag packaged fruits followed by non-perforated LDPE packed ones maintain their TSS value better than the control under cold storage condition whereas non-perforated LDPE packed fruits followed by perforated LDPE packed ones maintained their TSS value better than control under ambient condition. TSS of fruits kept in perforated and non-perforated packages and stored in the cold storage increased slowly and reached their maximum on $30^{\text {th }}$ day of storage. Slow increment in TSS of fruits in non-perforated plastic package during storage could be due to production of higher levels of $\mathrm{CO}_{2}$, which may lead to less physiological processes and slow ripening of fruits.

Those fruits packaged using perforated plastic and stored under ambient conditions attain their maximum TSS on day 15. On the other hand, non-perforated packaged fruits stored at ambient conditions reached their maximum TSS on day 20. Slight rises in the TSS levels were observed under both cold and ambient storage conditions regardless of the packaging types. However, the TSS of peach fruits was maintained at lower level in the cold storage than under ambient condition. High temperature enhances climacteric respiration, which leads to shorter shelf life of fruits [42]. Slow changes in TSS of fruits stored in the cold storage is in agreement with the findings of Aliye, et al. [43] for mango and Hirut, et al. [44] for tomato. This may be partly attributed to lower temperature and higher relative humidity maintained in the cold storage that could have resulted in slow conversion of starch in to water soluble sugars [42].

In general, packing of peach fruits in plastic bags combined with cold storage showed better maintenance of the TSS towards the end of storage time. In agreement with this result, increases occurred in TSS values with the prolonging storage period with MAP storage of peach and nectarine cultivars [45]. The changes reputed in TSS determined during the storage of fruits in a study on MAP of different peach and nectarine cultivars carried out by Agar, et al. [46] and Fernandez and Artes [47] support the results obtained from this study. The possible atmospheric modification, that is, reduced $\mathrm{O}_{2}$ and increased $\mathrm{CO}_{2}$ created in the package, combined with lower temperature in the cold storage might have delayed ripening of the fruits as a result of reduced respiration rate [36]. Hence, packed fruits do not rapidly deplete their soluble solids as those of the control fruits as observed in this study.

\section{4. $p H$}

The $\mathrm{pH}$ values of peach fruit genotypes subjected to different packaging and storage treatments for 30 days displayed in Table 3. Its values varied from 3.72-3.97 when stored in the cold storage and from 3.74-3.96 when stored under ambient conditions. Under both storage conditions, packed fruits had higher pH values compared to their respective control treatments. On day 5, cultivar 90-19H packed in non-perforated plastic and stored at ambient had the highest $\mathrm{pH}$ value (3.91). 
Table-2. Influence of plastic packing and storage condition on TSS of peach genotypes during 30 days of storage.

\begin{tabular}{|c|c|c|c|c|c|c|c|c|c|}
\hline \multicolumn{3}{|c|}{ Treatments } & & \multicolumn{6}{|c|}{ Storage period(days) } \\
\hline Variety & $\begin{array}{c}\text { Plastic } \\
\text { Packaging }\end{array}$ & Storage & $\bar{O}$ & 5 & 10 & 15 & 20 & 25 & 30 \\
\hline \multirow[t]{6}{*}{ Tropic Beauty } & \multirow[t]{2}{*}{ Perforated } & Cold & $8.46^{\mathrm{a}}$ & $9.07^{\mathrm{f}-\mathrm{j}}$ & $9.40^{\text {hi }}$ & $10.00^{\text {fg }}$ & $10.87^{\mathrm{e}}$ & $11.00^{\mathrm{d}}$ & $11.13^{\mathrm{d}}$ \\
\hline & & Ambient & $8.23^{\mathrm{a}}$ & $8.67^{\mathrm{ij}}$ & $9.07^{\mathrm{i}}$ & $10.27^{\mathrm{fg}}$ & - & - & - \\
\hline & \multirow{2}{*}{$\begin{array}{c}\text { Non- } \\
\text { perforated }\end{array}$} & Cold & $8.26^{\mathrm{a}}$ & $8.47^{\mathrm{ij}}$ & $8.73^{\mathrm{i}}$ & $9.73^{\mathrm{g}}$ & $10.13^{\mathrm{f}}$ & $10.60^{\mathrm{e}}$ & $10.73^{\mathrm{e}}$ \\
\hline & & Ambient & $8.40^{\mathrm{a}}$ & $8.33^{j}$ & $9.47^{\mathrm{hi}}$ & $9.80^{\mathrm{g}}$ & $10.40^{\mathrm{ef}}$ & - & - \\
\hline & \multirow{2}{*}{$\begin{array}{c}\text { Non- } \\
\text { packaged }\end{array}$} & Cold & $8.60^{\mathrm{a}}$ & $9.2 \mathrm{O}^{\mathrm{f}-\mathrm{j}}$ & $9.53^{\text {hi }}$ & - & - & - & - \\
\hline & & Ambient & $8.80^{\mathrm{a}}$ & $9.27^{\mathrm{f}-\mathrm{i}}$ & - & - & - & - & - \\
\hline \multirow[t]{6}{*}{$90-19 \mathrm{H}$} & \multirow[t]{2}{*}{ Perforated } & Cold & $8.46^{\mathrm{a}}$ & $8.30^{j}$ & $9.27^{\mathrm{i}}$ & $10.40^{\mathrm{fg}}$ & $10.80^{\mathrm{e}}$ & $10.93^{\mathrm{de}}$ & $11.07^{\mathrm{de}}$ \\
\hline & & Ambient & $8.33^{\mathrm{a}}$ & $8.73^{\text {hij }}$ & $10.10 \mathrm{gh}$ & $10.40^{\mathrm{fg}}$ & - & - & - \\
\hline & \multirow{2}{*}{$\begin{array}{c}\text { Non- } \\
\text { perforated }\end{array}$} & Cold & $8.46^{\mathrm{a}}$ & $8.80^{\text {hij }}$ & $9.27^{\mathrm{i}}$ & $10.07^{\mathrm{fg}}$ & $10.80^{\mathrm{e}}$ & $10.87^{\mathrm{de}}$ & $11.00^{\text {de }}$ \\
\hline & & Ambient & $8.66^{\mathrm{a}}$ & $8.93^{g-j}$ & $9.27^{\mathrm{i}}$ & $10.53^{\mathrm{f}}$ & $10.87^{\mathrm{e}}$ & - & - \\
\hline & \multirow{2}{*}{$\begin{array}{c}\text { Non- } \\
\text { packaged }\end{array}$} & Cold & $8.46^{\mathrm{a}}$ & $9.97 \mathrm{def}$ & $10.53^{\mathrm{fg}}$ & - & - & - & - \\
\hline & & Ambient & $8.66^{\mathrm{a}}$ & $9.33^{\mathrm{f}-\mathrm{i}}$ & - & - & - & - & - \\
\hline \multirow[t]{6}{*}{ Bonnigold } & \multirow[t]{2}{*}{ Perforated } & Cold & $8.66^{\mathrm{a}}$ & $9.80^{\mathrm{d}-\mathrm{g}}$ & $11.17^{\mathrm{c}-\mathrm{f}}$ & $12.87^{\mathrm{a}}$ & $12.93^{\mathrm{a}}$ & $13.13^{\mathrm{a}}$ & $13.33^{\mathrm{a}}$ \\
\hline & & Ambient & $8.4 \mathrm{O}^{\mathrm{a}}$ & $10.53^{\mathrm{cd}}$ & $11.2 \mathrm{O}^{\mathrm{c}-\mathrm{f}}$ & $11.53^{\text {cde }}$ & - & - & - \\
\hline & \multirow{2}{*}{$\begin{array}{c}\text { Non- } \\
\text { perforated }\end{array}$} & Cold & $8.46^{\mathrm{a}}$ & $10.33^{\mathrm{de}}$ & $10.80^{\mathrm{d}-\mathrm{g}}$ & $12.33^{\mathrm{ab}}$ & $12.47^{\mathrm{ab}}$ & $12.67^{\mathrm{b}}$ & $12.87^{\mathrm{b}}$ \\
\hline & & Ambient & $8.73^{\mathrm{a}}$ & $9.37^{\mathrm{fg}}$ & $11.53^{\mathrm{dc}}$ & $12.00^{\text {bcd }}$ & $12.2 \mathrm{O}^{\mathrm{bc}}$ & - & - \\
\hline & \multirow{2}{*}{$\begin{array}{c}\text { Non- } \\
\text { packaged }\end{array}$} & Cold & $8.5 \mathrm{O}^{\mathrm{a}}$ & $11.30^{\mathrm{bc}}$ & $13.33^{\mathrm{a}}$ & - & - & - & - \\
\hline & & Ambient & $8.40^{\mathrm{a}}$ & $11.33^{\mathrm{bc}}$ & - & - & - & - & - \\
\hline \multirow[t]{6}{*}{ Early Grande } & \multirow[t]{2}{*}{ Perforated } & Cold & $8.33^{\mathrm{a}}$ & $10.67^{\mathrm{cd}}$ & $11.40^{\text {cde }}$ & $12.13^{\mathrm{bc}}$ & $12.40^{\mathrm{ab}}$ & $12.53^{\mathrm{b}}$ & $12.67^{\mathrm{b}}$ \\
\hline & & Ambient & $8.86^{\mathrm{a}}$ & $11.70^{\mathrm{ab}}$ & $11.97^{\mathrm{bc}}$ & $12.40^{\mathrm{ab}}$ & - & - & - \\
\hline & \multirow{2}{*}{$\begin{array}{c}\text { Non- } \\
\text { perforated }\end{array}$} & Cold & $8.36^{\mathrm{a}}$ & $10.33^{\mathrm{de}}$ & $10.67^{\text {efg }}$ & $11.27^{\mathrm{e}}$ & $11.47^{\mathrm{d}}$ & $11.67^{\mathrm{c}}$ & $11.93^{\mathrm{c}}$ \\
\hline & & Ambient & $8.40^{\mathrm{a}}$ & $9.60^{\mathrm{e}-\mathrm{h}}$ & $10.80^{\mathrm{d}-\mathrm{g}}$ & $11.33^{\mathrm{de}}$ & $11.67^{\mathrm{cd}}$ & - & - \\
\hline & \multirow{2}{*}{$\begin{array}{c}\text { Non- } \\
\text { packaged }\end{array}$} & Cold & $8.53^{\mathrm{a}}$ & $12.27^{\mathrm{a}}$ & $12.73^{\mathrm{ab}}$ & - & - & - & - \\
\hline & & Ambient & $8.40^{\mathrm{a}}$ & $10.60^{\mathrm{cd}}$ & - & - & - & - & - \\
\hline \multicolumn{3}{|c|}{$\mathrm{SE} \pm$} & 0.27 & 0.33 & 0.28 & 0.25 & 0.20 & 0.13 & 0.12 \\
\hline \multicolumn{3}{|c|}{ CV (\%) } & 5.62 & 5.75 & 4.63 & 3.90 & 3.09 & 1.92 & 1.82 \\
\hline
\end{tabular}

Note: $\mathrm{SE}=$ Standard error, $\mathrm{CV}=$ Coefficient of variation, means with different letters in a column indicate significant differences at $\mathrm{p}=0.05$.

Table-3. Influence of plastic packing and storage condition on $\mathrm{pH}$ of peach genotypes during 30 days of storage.

\begin{tabular}{|c|c|c|c|c|c|c|c|c|c|}
\hline \multicolumn{3}{|c|}{ Treatments } & & \multicolumn{6}{|c|}{ Storage period(days) } \\
\hline Variety & $\begin{array}{c}\text { Plastic } \\
\text { Packaging }\end{array}$ & Storage & $\mathrm{O}$ & 5 & 10 & 15 & 20 & 25 & 30 \\
\hline \multirow[t]{6}{*}{ Tropic Beauty } & \multirow[t]{2}{*}{ Perforated } & Cold & $3.72^{\mathrm{a}}$ & $3.81^{\mathrm{de}}$ & $3.84^{\mathrm{f}-\mathrm{i}}$ & $3.85^{\mathrm{d}}$ & $3.89^{\mathrm{bc}}$ & $3.91^{\mathrm{bc}}$ & $3.95^{\mathrm{b}}$ \\
\hline & & Ambient & $3.71^{\mathrm{a}}$ & $3.81^{\mathrm{de}}$ & $3.82^{\text {hi }}$ & $3.84^{\mathrm{de}}$ & - & - & - \\
\hline & \multirow{2}{*}{$\begin{array}{c}\text { Non- } \\
\text { perforated }\end{array}$} & Cold & $3.72^{\mathrm{a}}$ & $3.85^{\mathrm{b}}$ & $3.89^{\mathrm{c}}$ & $3.90^{\mathrm{b}}$ & $3.90^{\mathrm{b}}$ & $3.92^{\mathrm{ab}}$ & $3.95^{\mathrm{b}}$ \\
\hline & & Ambient & $3.71^{\mathrm{a}}$ & $3.91^{\mathrm{a}}$ & $3.92^{\mathrm{a}}$ & $3.94^{\mathrm{a}}$ & $3.95^{\mathrm{a}}$ & - & - \\
\hline & \multirow{2}{*}{$\begin{array}{c}\text { Non- } \\
\text { packaged }\end{array}$} & Cold & $3.71^{\mathrm{a}}$ & $3.84^{\mathrm{b}}$ & $3.86^{\mathrm{def}}$ & - & - & - & - \\
\hline & & Ambient & $3.71^{\mathrm{a}}$ & $3.82^{\text {cde }}$ & - & - & - & - & - \\
\hline \multirow[t]{6}{*}{$9 \mathrm{O}-19 \mathrm{H}$} & \multirow[t]{2}{*}{ Perforated } & Cold & $3.71^{\mathrm{a}}$ & $3.73^{\mathrm{ij}}$ & $3.77^{j}$ & $3.85^{\mathrm{d}}$ & $3.87^{\mathrm{cd}}$ & $3.92^{\mathrm{ab}}$ & $3.95^{\mathrm{b}}$ \\
\hline & & Ambient & $3.71^{\mathrm{a}}$ & $3.85^{\mathrm{b}}$ & $3.83^{\text {ghi }}$ & $3.93^{\mathrm{a}}$ & - & - & - \\
\hline & \multirow{2}{*}{$\begin{array}{c}\text { Non- } \\
\text { perforated }\end{array}$} & Cold & $3.72^{\mathrm{a}}$ & $3.86^{\mathrm{b}}$ & $3.89^{\mathrm{bc}}$ & $3.90^{\mathrm{b}}$ & $3.91^{\mathrm{b}}$ & $3.94^{\mathrm{a}}$ & $3.97^{\mathrm{a}}$ \\
\hline & & Ambient & $3.71^{\mathrm{a}}$ & $3.91^{\mathrm{a}}$ & $3.91^{\mathrm{ab}}$ & $3.94^{\mathrm{a}}$ & $3.96^{\mathrm{a}}$ & - & - \\
\hline & \multirow{2}{*}{$\begin{array}{c}\text { Non- } \\
\text { packaged }\end{array}$} & Cold & $3.72^{\mathrm{a}}$ & $3.84^{\mathrm{bc}}$ & $3.85^{\text {efg }}$ & - & - & - & - \\
\hline & & Ambient & $3.71^{\mathrm{a}}$ & $3.79^{\mathrm{fg}}$ & - & - & - & - & - \\
\hline \multirow[t]{6}{*}{ Bonnigold } & \multirow[t]{2}{*}{ Perforated } & Cold & $3.71^{\mathrm{a}}$ & $3.81^{\mathrm{de}}$ & $3.84^{\mathrm{f}-\mathrm{i}}$ & $3.86^{\mathrm{cd}}$ & $3.89^{\mathrm{bc}}$ & $3.90^{\mathrm{bc}}$ & $3.93^{\mathrm{c}}$ \\
\hline & & Ambient & $3.72^{\mathrm{a}}$ & $3.74^{\mathrm{hi}}$ & $3.82^{\text {hi }}$ & $3.83^{\mathrm{e}}$ & - & - & - \\
\hline & \multirow{2}{*}{$\begin{array}{c}\text { Non- } \\
\text { perforated }\end{array}$} & Cold & $3.71^{\mathrm{a}}$ & $3.72^{j}$ & $3.77^{j}$ & $3.84^{\mathrm{de}}$ & $3.86^{\mathrm{d}}$ & $3.89^{c}$ & $3.93^{\mathrm{c}}$ \\
\hline & & Ambient & $3.71^{\mathrm{a}}$ & $3.81^{\mathrm{de}}$ & $3.85^{\mathrm{e}-\mathrm{h}}$ & $3.88^{\mathrm{bc}}$ & $3.91^{\mathrm{b}}$ & - & - \\
\hline & \multirow{2}{*}{$\begin{array}{c}\text { Non- } \\
\text { packaged }\end{array}$} & Cold & $3.71^{\mathrm{a}}$ & $3.75^{\text {hi }}$ & $3.78^{j}$ & - & - & - & - \\
\hline & & Ambient & $3.71^{\mathrm{a}}$ & $3.81^{\mathrm{de}}$ & - & - & - & - & - \\
\hline \multirow[t]{6}{*}{ Early Grande } & \multirow[t]{2}{*}{ Perforated } & Cold & $3.70^{\mathrm{a}}$ & $3.81^{\mathrm{de}}$ & $3.83^{\text {ghi }}$ & $3.88^{\mathrm{bc}}$ & $3.89^{\mathrm{bc}}$ & $3.91^{\mathrm{bc}}$ & $3.93^{\mathrm{c}}$ \\
\hline & & Ambient & $3.71^{\mathrm{a}}$ & $3.76^{\mathrm{h}}$ & $3.82^{\mathrm{hi}}$ & $3.89^{\mathrm{b}}$ & - & - & - \\
\hline & \multirow{2}{*}{$\begin{array}{c}\text { Non- } \\
\text { perforated }\end{array}$} & Cold & $3.71^{\mathrm{a}}$ & $3.83^{\mathrm{bcd}}$ & $3.88^{\mathrm{cd}}$ & $3.89^{\mathrm{bc}}$ & $3.90^{\mathrm{bc}}$ & $3.91^{\mathrm{bc}}$ & $3.93^{\mathrm{c}}$ \\
\hline & & Ambient & $3.71^{\mathrm{a}}$ & $3.76^{\mathrm{gh}}$ & $3.93^{\mathrm{a}}$ & $3.95^{\mathrm{a}}$ & $3.96^{\mathrm{a}}$ & - & - \\
\hline & \multirow{2}{*}{$\begin{array}{c}\text { Non- } \\
\text { packaged }\end{array}$} & Cold & $3.71^{\mathrm{a}}$ & $3.77^{\text {gh }}$ & $3.87^{\text {cde }}$ & - & - & - & - \\
\hline & & Ambient & $3.7 \mathrm{O}^{\mathrm{a}}$ & $3.81^{\mathrm{de}}$ & - & - & - & - & - \\
\hline \multicolumn{3}{|c|}{$\mathrm{SE} \pm$} & 0.01 & 0.01 & 0.01 & 0.01 & 0.01 & 0.01 & 0.01 \\
\hline \multicolumn{3}{|c|}{ CV (\%) } & 0.42 & 0.42 & 0.37 & 0.41 & 0.37 & 0.33 & 0.24 \\
\hline
\end{tabular}

Note: $\mathrm{SE}=$ Standard error, $\mathrm{CV}=$ Coefficient of variation, means with different letters in a column indicate significant differences at $\mathrm{p}=0.05$.

On day 10 of storage time, Early Grande packed in non-perforated plastic and kept at ambient had the highest $\mathrm{pH}$ (3.93). On day 30, genotype 90-19H packaged in non-perforated plastic and stored in cold storage had the highest $\mathrm{pH}$ value (3.97) while the least value (3.93) was recorded with Bonnigold and Early Grande that were packed in both perforated and non-perforated plastic.

The higher $\mathrm{pH}$ of fruits under ambient storage conditions at initial stage could be associated with the breakdown of acids and sugar in respiration process at faster rate under ambient than in the cold condition [48]. Hence, lowering the storage temperature can reduce respiration rate and delay senescence of peach fruits. Relatively lower $\mathrm{pH}$ values of packed fruits could be explained by reduced respiration rate in the package. Reduced $\mathrm{O}_{2}$ and increased $\mathrm{CO}_{2}$ which could be created as a result of respiration could delay the rate of respiration in the package and keep $\mathrm{pH}$ values low [36]. The rate of use of acids as respiratory substrates may increase at higher temperature and result in depletion of acid content of fruits stored at ambient condition [48]. $\mathrm{pH}$ values of peach 
fruits, generally, increased with ripening of the fruits and this could be attributed to the fact that fruits with ripening process diminish its predominant malic acid [49]. The increase in $\mathrm{pH}$ value of peach with advance in storage time is in agreement with the findings of Agozzino, et al. [50].

\subsection{Titratable Acidity}

The interaction between packing materials, genotypes and storage conditions had highly significant $(\mathrm{p} \leq 0.001)$ effect on the titratable acidity of peach fruits Table 4. Titratable acidity of the sample fruits, irrespective of the treatments, decreased linearly throughout the storage period, as opposed to $\mathrm{pH}$ value which showed increasing trend. The TA value varied from $0.82 \%$ in $90-19 \mathrm{H}$ genotype packed in plastic and stored in cold storage on day 30 to $1.21 \%$ in Early Grande cultivar packed in perforated plastic and stored under ambient condition on day 5 . This trend is in agreement with the findings of Pongener, et al. [51] that the titratable acidity of peach fruits packed under polythene films showed a linear declining trend with the advancement of storage period which could be due to its use by the cells as a respiration substrate [49]. On day 5 and 10 of storage, the TA of Early Grande variety under perforated plastic package and stored at ambient condition was significantly higher than TA values in all other treatments.

Table-4. Influence of plastic packaging and storage condition on TA of peach genotypes during 30 days of storage.

\begin{tabular}{|c|c|c|c|c|c|c|c|c|c|}
\hline \multicolumn{3}{|c|}{ Treatments } & & \multicolumn{6}{|c|}{ Storage period(days) } \\
\hline Variety & Plastic Packaging & Storage & $\mathrm{O}$ & 5 & 10 & 15 & 20 & 25 & 30 \\
\hline \multirow[t]{6}{*}{ Tropic Beauty } & \multirow[t]{2}{*}{ Perforated } & Cold & $0.72^{\mathrm{a}}$ & $1.09^{\text {cde }}$ & $1.06^{\mathrm{cd}}$ & $1.02^{\mathrm{cd}}$ & $0.98^{\mathrm{ab}}$ & $0.93^{\mathrm{ab}}$ & $0.88^{\mathrm{bc}}$ \\
\hline & & Ambient & $0.73^{\mathrm{a}}$ & $1.11^{\mathrm{cd}}$ & $1.05^{\mathrm{cd}}$ & $0.99^{\mathrm{cd}}$ & - & - & - \\
\hline & \multirow[t]{2}{*}{ Non-perforated } & Cold & $0.72^{\mathrm{a}}$ & $1.06^{\mathrm{def}}$ & $1.02^{\mathrm{cd}}$ & $0.99^{\mathrm{cd}}$ & $0.96^{\mathrm{b}}$ & $0.94^{\mathrm{ab}}$ & $0.89^{\mathrm{bc}}$ \\
\hline & & Ambient & $0.73^{\mathrm{a}}$ & $1.04^{\mathrm{ef}}$ & $1.01^{\mathrm{d}}$ & $0.96^{\text {def }}$ & $0.89^{\mathrm{c}}$ & - & - \\
\hline & \multirow[t]{2}{*}{ Non-packaged } & Cold & $0.73^{\mathrm{a}}$ & $1.10^{\text {cde }}$ & $1.07^{\mathrm{cd}}$ & - & - & - & - \\
\hline & & Ambient & $0.73^{\mathrm{a}}$ & $0.82^{j}$ & - & - & - & - & - \\
\hline \multirow[t]{6}{*}{$90-19 \mathrm{H}$} & \multirow[t]{2}{*}{ Perforated } & Cold & $0.73^{\mathrm{a}}$ & $1.03^{\mathrm{fg}}$ & $1.00^{\mathrm{d}}$ & $0.93^{\text {efg }}$ & $0.87^{\mathrm{c}}$ & $0.84^{\mathrm{c}}$ & $0.82^{\mathrm{d}}$ \\
\hline & & Ambient & $0.72^{\mathrm{a}}$ & $0.96^{\mathrm{h}}$ & $0.89^{\mathrm{e}}$ & $0.89^{g}$ & - & - & - \\
\hline & \multirow[t]{2}{*}{ Non-perforated } & Cold & $0.73^{\mathrm{a}}$ & $0.95^{\mathrm{h}}$ & $0.87^{\mathrm{e}}$ & $0.88^{g}$ & $0.86^{\mathrm{c}}$ & $0.84^{\mathrm{c}}$ & $0.82^{\mathrm{d}}$ \\
\hline & & Ambient & $0.72^{\mathrm{a}}$ & $0.96^{\mathrm{h}}$ & $0.90^{\mathrm{e}}$ & $0.89^{g}$ & $0.86^{\mathrm{c}}$ & - & - \\
\hline & \multirow[t]{2}{*}{ Non-packaged } & Cold & $0.72^{\mathrm{a}}$ & $1.09^{\text {cde }}$ & $1.05^{\mathrm{cd}}$ & - & - & - & - \\
\hline & & Ambient & $0.72^{\mathrm{a}}$ & $0.95^{\mathrm{h}}$ & - & - & - & - & - \\
\hline \multirow[t]{6}{*}{ Bonnigold } & \multirow[t]{2}{*}{ Perforated } & Cold & $0.72^{\mathrm{a}}$ & $1.17^{\mathrm{ab}}$ & $1.16^{\mathrm{ab}}$ & $1.12^{\mathrm{a}}$ & $1.02^{\mathrm{a}}$ & $0.96^{\mathrm{a}}$ & $0.93^{\mathrm{a}}$ \\
\hline & & Ambient & $0.72^{\mathrm{a}}$ & $1.07^{\mathrm{c}-\mathrm{f}}$ & $1.05^{\mathrm{cd}}$ & $1.05^{\mathrm{bc}}$ & - & - & - \\
\hline & \multirow[t]{2}{*}{ Non-perforated } & Cold & $0.73^{\mathrm{a}}$ & $1.06^{\mathrm{def}}$ & $1.04^{\mathrm{cd}}$ & $1.01^{\mathrm{cd}}$ & $0.99^{\mathrm{ab}}$ & $0.95^{\mathrm{a}}$ & $0.92^{\mathrm{ab}}$ \\
\hline & & Ambient & $0.73^{\mathrm{a}}$ & $1.12^{\mathrm{bc}}$ & $1.09^{\mathrm{bc}}$ & $1.05^{\mathrm{bc}}$ & $1.01^{\mathrm{a}}$ & - & - \\
\hline & \multirow[t]{2}{*}{ Non-packaged } & Cold & $0.72^{\mathrm{a}}$ & $1.04^{\mathrm{ef}}$ & $1.02^{\mathrm{cd}}$ & - & - & - & - \\
\hline & & Ambient & $0.72^{\mathrm{a}}$ & $1.03^{\mathrm{fg}}$ & - & - & - & - & - \\
\hline \multirow[t]{6}{*}{ Early Grande } & \multirow[t]{2}{*}{ Perforated } & Cold & $0.72^{\mathrm{a}}$ & $1.07^{\mathrm{c}-\mathrm{f}}$ & $1.04^{\mathrm{cd}}$ & $1.04^{\mathrm{bc}}$ & $0.99^{\mathrm{ab}}$ & $0.92^{\mathrm{ab}}$ & $0.88^{\mathrm{bc}}$ \\
\hline & & Ambient & $0.73^{\mathrm{a}}$ & $1.21^{\mathrm{a}}$ & $1.21^{\mathrm{a}}$ & $1.10^{\mathrm{ab}}$ & - & - & - \\
\hline & \multirow[t]{2}{*}{ Non-perforated } & Cold & $0.72^{\mathrm{a}}$ & $0.96^{\mathrm{h}}$ & $0.91^{\mathrm{e}}$ & $0.99^{\mathrm{cd}}$ & $0.96^{\mathrm{b}}$ & $0.90^{\mathrm{b}}$ & $0.84^{\mathrm{cd}}$ \\
\hline & & Ambient & $0.72^{\mathrm{a}}$ & $0.97^{\text {gh }}$ & $0.91^{\mathrm{e}}$ & $0.92^{\mathrm{fg}}$ & $0.90^{\mathrm{c}}$ & - & - \\
\hline & \multirow[t]{2}{*}{ Non-packaged } & Cold & $0.73^{\mathrm{a}}$ & $1.12^{\mathrm{bcd}}$ & $1.09^{\mathrm{bc}}$ & - & - & - & - \\
\hline & & Ambient & $0.73^{\mathrm{a}}$ & $0.88^{\mathrm{i}}$ & - & - & - & - & - \\
\hline \multicolumn{3}{|c|}{$\mathrm{SE} \pm$} & 0.01 & 0.02 & 0.03 & 0.02 & 0.01 & 0.02 & 0.02 \\
\hline \multicolumn{3}{|c|}{$\mathrm{CV}(\%)$} & 1.59 & 3.50 & 4.55 & 3.99 & 2.57 & 3.36 & 3.02 \\
\hline
\end{tabular}

Perforated plastic packaged fruits of Bonnigold variety in the cold storage had higher TA values starting from day 15 to 30 , indicating varietal difference and effect of storage condition on this parameter. Likewise, nonperforated plastic packaging tended to have TA values relatively lower than perforated packaged fruits and the control under their respective storage conditions and varieties.

Previous report also shows that LDPE non-perforated packed fruits could maintain a higher level of acidity [35]. It might be due to reduced respiration rate in the later stage of storage as affected by film permeability to atmospheric gas. The titratable acidity of fruits was an indicator of potential storage quality and declined gradually over the storage period [52].

The lower TA value of polyethylene bag packed fruits in the cold storage could be explained by the reduced rate respiration which results in slow use of organic acids as a substrate of respiration. The higher loss of TA in control fruits could be due to depletion of organic acids as a result of relatively faster respiration and ripening rate of fruits at ambient storage Zerbini [53]. Kader [54] the atmospheric modification created when fruits are packed with bags may delay respiration and consumption of respiration substrates such as organic acids and sugars are reduced. Consequently, as the fruit respires, the $\mathrm{O}_{2}$ level decreases and the $\mathrm{CO}_{2}$ level increases in the bags [54] . Under these atmospheric conditions, the respiration rate of the fruit decrease which is helpful since high acidity in fruit has been suggested to contribute in part to the flavor retention of ripened fruit [55]. Generally, TA content of peach fruit had been maintained higher in the cold storage compared with ambient condition.

\subsection{TSS/TA (Ripening Index)}

The interaction effect between packing materials and storage conditions had significant $(p \leq 0.05)$ effect on the ripening index of peach genotypes Table 5. The TSS/TA values of peach fruits showed an increase with storage time, which also varied with genotype and storage condition. TSS/TA ratio has important roles to play as parameters that can be used to tell maturity of peach fruit, especially for small-scale growers [56]. The ripening index is important for fruit flavor with lower values makes fruits more favorable [57].

On day 5, Early Grande fruits without package and stored at ambient had the highest TSS/TA ratio (12.09). Compared to rest of the cultivars, Tropic Beauty recorded relatively lower TSS/TA values except in non-packaged 
ones kept at ambient storage. On the $10^{\text {th }}$ day of storage, non-packaged fruits of Bonnigold kept in cold storage resulted in the highest TSS/TA value (13.03).

\begin{tabular}{|c|c|c|c|c|c|c|c|c|c|}
\hline \multicolumn{3}{|c|}{ Treatments } & \multirow[b]{2}{*}{$\mathbf{0}$} & \multicolumn{6}{|c|}{ Storage period(days) } \\
\hline Variety & $\begin{array}{c}\text { Plastic } \\
\text { Packaging }\end{array}$ & Storage & & 5 & 10 & 15 & 20 & 25 & 30 \\
\hline \multirow{6}{*}{$\begin{array}{l}\text { Tropic } \\
\text { Beauty }\end{array}$} & \multirow[t]{2}{*}{ Perforated } & Cold & $10.9^{\mathrm{b}}$ & $8.30^{\mathrm{gh}}$ & $8.84^{\mathrm{hi}}$ & $9.84^{\mathrm{f}}$ & $11.13^{\mathrm{ef}}$ & $11.79^{\mathrm{b}}$ & $12.65^{\mathrm{c}}$ \\
\hline & & Ambient & $11.4^{\mathrm{ab}}$ & $7.82^{\mathrm{h}}$ & $8.66^{\mathrm{i}}$ & $10.33^{\mathrm{def}}$ & - & - & - \\
\hline & \multirow{2}{*}{$\begin{array}{c}\text { Non- } \\
\text { perforated }\end{array}$} & Cold & $12.0^{\mathrm{ab}}$ & $8.01^{\mathrm{h}}$ & $8.62^{\mathrm{i}}$ & $9.83^{\mathrm{f}}$ & $10.53^{\mathrm{f}}$ & $11.30^{\mathrm{b}}$ & $12.12^{\mathrm{c}}$ \\
\hline & & Ambient & $11.6^{\mathrm{ab}}$ & $7.99^{\mathrm{h}}$ & $9.41^{\text {ghi }}$ & $10.22^{\mathrm{ef}}$ & $11.73^{\mathrm{de}}$ & - & - \\
\hline & \multirow{2}{*}{$\begin{array}{c}\text { Non- } \\
\text { packaged }\end{array}$} & Cold & $11.9^{\mathrm{ab}}$ & $8.37^{\mathrm{gh}}$ & $8.95^{\mathrm{hi}}$ & - & - & - & - \\
\hline & & Ambient & $12.0^{\mathrm{ab}}$ & $11.37^{\mathrm{ab}}$ & - & - & - & - & - \\
\hline \multirow[t]{6}{*}{$90-19 \mathrm{H}$} & \multirow[t]{2}{*}{ Perforated } & Cold & $11.5^{\mathrm{ab}}$ & $8.10^{\mathrm{h}}$ & $9.27^{\text {ghi }}$ & $11.25^{\mathrm{bcd}}$ & $12.47^{\mathrm{abc}}$ & $12.98^{\mathrm{a}}$ & $13.51^{\mathrm{b}}$ \\
\hline & & Ambient & $11.4^{\mathrm{ab}}$ & $9.11^{\mathrm{fg}}$ & $11.32^{\mathrm{bc}}$ & $11.69^{\mathrm{abc}}$ & - & - & - \\
\hline & \multirow{2}{*}{$\begin{array}{c}\text { Non- } \\
\text { perforated }\end{array}$} & Cold & $11.5^{\mathrm{ab}}$ & $9.31^{\mathrm{fg}}$ & $10.70^{\text {cde }}$ & $11.44^{\mathrm{bc}}$ & $12.52^{\mathrm{abc}}$ & $12.94^{\mathrm{a}}$ & $13.47^{\mathrm{b}}$ \\
\hline & & Ambient & $11.9^{\mathrm{ab}}$ & $9.32^{\mathrm{fg}}$ & $10.34^{\text {def }}$ & $11.88^{\mathrm{abc}}$ & $12.59^{\mathrm{abc}}$ & - & - \\
\hline & \multirow{2}{*}{$\begin{array}{c}\text { Non- } \\
\text { packaged }\end{array}$} & Cold & $11.6^{\mathrm{ab}}$ & $9.14^{\mathrm{fg}}$ & $10.09^{\text {defg }}$ & - & - & - & - \\
\hline & & Ambient & $11.9^{\mathrm{ab}}$ & $9.80^{\mathrm{ef}}$ & - & - & - & - & - \\
\hline \multirow[t]{6}{*}{ Bonnigold } & \multirow[t]{2}{*}{ Perforated } & Cold & $11.8^{\mathrm{ab}}$ & $8.38^{\mathrm{gh}}$ & $9.61^{\text {fgh }}$ & $11.49^{\text {abc }}$ & $12.72^{\mathrm{ab}}$ & $13.54^{\mathrm{a}}$ & $14.29^{\mathrm{a}}$ \\
\hline & & Ambient & $12.2^{\mathrm{a}}$ & $9.84^{\text {def }}$ & $10.73^{\text {cde }}$ & $11.05^{\text {cde }}$ & - & - & - \\
\hline & \multirow{2}{*}{$\begin{array}{c}\text { Non- } \\
\text { perforated }\end{array}$} & Cold & $11.7^{\mathrm{ab}}$ & $9.72^{f}$ & $10.42^{\text {def }}$ & $12.17^{\mathrm{ab}}$ & $12.56^{\mathrm{abc}}$ & $13.29^{\mathrm{a}}$ & $13.99^{\mathrm{ab}}$ \\
\hline & & Ambient & $11.9^{\mathrm{ab}}$ & $8.34^{\text {gh }}$ & $10.60^{\text {cde }}$ & $11.45^{\mathrm{bc}}$ & $12.08^{\mathrm{bcd}}$ & - & - \\
\hline & \multirow{2}{*}{$\begin{array}{c}\text { Non- } \\
\text { packaged }\end{array}$} & Cold & $11.6^{\mathrm{ab}}$ & $10.83^{\text {bcd }}$ & $13.03^{\mathrm{a}}$ & - & - & - & - \\
\hline & & Ambient & $11.6^{\mathrm{ab}}$ & $11.01^{\mathrm{bc}}$ & - & - & - & - & - \\
\hline \multirow{6}{*}{$\begin{array}{c}\text { Early } \\
\text { Grande }\end{array}$} & \multirow[t]{2}{*}{ Perforated } & Cold & $11.5^{\mathrm{ab}}$ & $10.01^{\mathrm{c}-\mathrm{f}}$ & $10.93^{\mathrm{bcd}}$ & $11.71^{\mathrm{abc}}$ & $12.48^{\mathrm{abc}}$ & $13.58^{\mathrm{a}}$ & $14.34^{\mathrm{a}}$ \\
\hline & & Ambient & $12.3^{\mathrm{a}}$ & $9.64^{\mathrm{f}}$ & $9.93^{\mathrm{efg}}$ & $11.31^{\mathrm{bc}}$ & - & - & - \\
\hline & \multirow{2}{*}{$\begin{array}{c}\text { Non- } \\
\text { perforated }\end{array}$} & Cold & $11.6^{\mathrm{ab}}$ & $10.77^{\mathrm{b}-\mathrm{e}}$ & $11.73^{\mathrm{b}}$ & $11.34^{\mathrm{bc}}$ & $11.90^{\mathrm{cd}}$ & $13.01^{\mathrm{a}}$ & $14.16^{\mathrm{ab}}$ \\
\hline & & Ambient & $11.5^{\mathrm{ab}}$ & $9.87^{\mathrm{def}}$ & $11.82^{\mathrm{b}}$ & $12.4 \mathrm{O}^{\mathrm{a}}$ & $12.99^{\mathrm{a}}$ & - & - \\
\hline & \multirow{2}{*}{$\begin{array}{c}\text { Non- } \\
\text { packaged }\end{array}$} & Cold & $11.7^{\mathrm{ab}}$ & $10.98^{\text {bc }}$ & $11.64^{\mathrm{b}}$ & - & - & - & - \\
\hline & & Ambient & $11.5^{\mathrm{ab}}$ & $12.09^{\mathrm{a}}$ & - & - & - & - & - \\
\hline \multicolumn{3}{|c|}{$\mathrm{SE} \pm$} & 0.39 & 0.36 & 0.39 & 0.32 & 0.24 & 0.24 & 0.24 \\
\hline \multicolumn{3}{|c|}{$\mathrm{CV}(\%)$} & 5.85 & 6.57 & 5.85 & 4.95 & 3.39 & 3.21 & 3.03 \\
\hline
\end{tabular}

Towards the end of storage, Bonnigold and Early Grande cultivars either in perforated or non-perforated plastics had higher TSS/TA values $(13.99-14.34)$ while the lowest was recorded in Tropic Beauty under similar storage condition. Generally, there were increasing trend of TSS/TA towards the end of storage time due to increased TSS and decreased TA for respective treatment under both storage conditions which was similar with the findings of Mkhathini, et al. [56]. Similarly, the decrease in $\mathrm{O}_{2}$ and elevated $\mathrm{CO}_{2}$ due to packaging delay the ripening [58] and resulted in lower TSS/Acid ratio [59]. The negative correlation between firmness and TSS/TA reflect an increase in TSS and decrease in TA with decreasing firmness upon ripening [59].

\subsection{Ascorbic Acid (Vitamin C)}

The interaction of packaging materials, genotypes and storage condition showed highly significant effect $(\mathrm{P} \leq 0.001)$ on the ascorbic acid (AA) content of peach fruit Table 6. Vit. C content of peach fruits varied from 11.446.44 $\mathrm{mg} 100^{-1} \mathrm{~g}$ which is in agreement with the range of $10.2-6.2 \mathrm{mg} 100^{-1} \mathrm{~g}$ AA reported by Mahajan, et al. [60]. Ascorbic acid content of the fruit generally showed linear decline during storage. Similar findings were also reported by Soliva-Fortuny and Martín-Belloso [52] in pear. On day 5 and 10 Bonnigold cultivar packaged in perforated plastic and kept at ambient had higher ascorbic acid content, which was at par with values of fruits of Early Grande cultivar kept in perforated plastic and stored in cold storage; After 15 days of storage, Bonnigold fruits with perforated plastic packages stored in cold store recorded higher ascorbic acid values, with the values from Early Grande being at par on day 15 and 20 and Bonnigold fruits in non-perforated fruits stored in cold store.

All treatments showed decreasing trend towards the end of the storage time both at cold and ambient conditions. Activities of oxidizing enzymes might reduce in packed fruits and stored in cold result in higher vitamin $\mathrm{C}$ retention up to last day of storage. Variation in vitamin $\mathrm{C}$ retention in different treatments might be due to genetic differences and levels of oxidation as affected by temperature and plastic permeability to environmental oxygen. Lower loss of ascorbic acid content might be due to low $\mathrm{O}_{2}$ permeability, which reduces respiration rate. During storage, higher temperature and oxygen favor activities of oxidizing enzymes like ascorbic acid oxidase, peroxidase, catalase and polyphenol oxidase to act on substrates of respiration, including acids, and hasten loss of ascorbic acid of the fruits [61].

The vitamin $\mathrm{C}$ content decreased rapidly which was higher under ambient than in the cold storage. As the storage time increased, the trend was changed. Peaches stored in the cold storage showed more vitamin $\mathrm{C}$ content than those stored under ambient conditions. Packed fruits maintain and showed higher Vitamin $\mathrm{C}$ content than the control. This might be due to reduced rate of respiration at lower temperature and inside the package that retards respiration and depletion of acids. Furthermore, the reduction in internal $\mathrm{O}_{2}$ and ethylene concentration might explain the maintenance of higher value of AA in packed fruits although there is delay in respiration and ripening $[54]$. 
Table -6. Influence of plastic packing and storage condition on vitamin C content of peach genotypes during 30 days of storage.

\begin{tabular}{|c|c|c|c|c|c|c|c|c|c|}
\hline \multicolumn{3}{|c|}{ Treatments } & & \multicolumn{6}{|c|}{ Storage period (days) } \\
\hline Variety & $\begin{array}{c}\text { Plastic } \\
\text { Packaging }\end{array}$ & Storage & $\mathbf{0}$ & 5 & 10 & 15 & 20 & 25 & 30 \\
\hline \multirow[t]{6}{*}{ Tropic Beauty } & \multirow[t]{2}{*}{ Perforated } & Cold & $9.44^{\mathrm{a}}$ & $9.11^{\text {ghi }}$ & $9.11^{\text {cde }}$ & $8.78^{\mathrm{ef}}$ & $8.44^{b}$ & $7.89^{\mathrm{de}}$ & $7.56^{\mathrm{de}}$ \\
\hline & & Ambient & $8.99^{\mathrm{a}}$ & $8.22^{\mathrm{ijk}}$ & $8.00^{\mathrm{fg}}$ & $7.33^{\mathrm{h}}$ & - & - & - \\
\hline & \multirow{2}{*}{$\begin{array}{c}\text { Non- } \\
\text { perforated }\end{array}$} & Cold & $9.55^{\mathrm{a}}$ & $9.22^{\text {fgh }}$ & $9.00^{\text {de }}$ & $8.78^{\mathrm{ef}}$ & $8.44^{b}$ & $7.89^{\text {de }}$ & $7.44^{\mathrm{de}}$ \\
\hline & & Ambient & $9.11^{\mathrm{a}}$ & $9.55^{\mathrm{d}-\mathrm{h}}$ & $9.11^{\text {cde }}$ & $8.56^{\mathrm{f}}$ & $7.44^{\mathrm{cd}}$ & - & - \\
\hline & \multirow{2}{*}{$\begin{array}{c}\text { Non- } \\
\text { packaged }\end{array}$} & Cold & $9.11^{\mathrm{a}}$ & $8.67^{\text {hij }}$ & $7.67^{\mathrm{g}}$ & - & - & - & - \\
\hline & & Ambient & $9.11^{\mathrm{a}}$ & $8.67^{\text {hij }}$ & - & - & - & - & - \\
\hline \multirow[t]{6}{*}{$9 \mathrm{O}-19 \mathrm{H}$} & \multirow[t]{2}{*}{ Perforated } & Cold & $9.11^{\mathrm{a}}$ & $7.66^{\mathrm{k}}$ & $7.67^{\mathrm{g}}$ & $7.44^{\text {gh }}$ & $7.22^{\mathrm{d}}$ & $6.67^{\mathrm{f}}$ & $6.44^{\mathrm{f}}$ \\
\hline & & Ambient & $8.89^{\mathrm{a}}$ & $8.22^{\mathrm{ijk}}$ & $7.89^{\mathrm{g}}$ & $7.44^{\text {gh }}$ & - & - & - \\
\hline & \multirow{2}{*}{$\begin{array}{c}\text { Non- } \\
\text { perforated }\end{array}$} & Cold & $8.99^{\mathrm{a}}$ & $8.78^{h i j}$ & $8.78^{\mathrm{ef}}$ & $8.44^{\mathrm{f}}$ & $7.89^{c}$ & $7.44^{\mathrm{e}}$ & $7.33^{\mathrm{e}}$ \\
\hline & & Ambient & $9.00^{\mathrm{a}}$ & $9.89^{\mathrm{c}-\mathrm{g}}$ & $9.33^{\text {cde }}$ & $9.33^{\mathrm{bcd}}$ & $8.44^{b}$ & - & - \\
\hline & \multirow{2}{*}{$\begin{array}{c}\text { Non- } \\
\text { packaged }\end{array}$} & Cold & $8.77^{\mathrm{a}}$ & $8.00 j^{k}$ & $7.44^{g}$ & - & - & - & - \\
\hline & & Ambient & $8.89^{\mathrm{a}}$ & $7.44^{k}$ & - & - & - & - & - \\
\hline \multirow[t]{6}{*}{ Bonnigold } & \multirow[t]{2}{*}{ Perforated } & Cold & $9.11^{\mathrm{a}}$ & $9.7^{\mathrm{c}-\mathrm{g}}$ & $9.67^{\mathrm{bcd}}$ & $9.89^{\mathrm{a}}$ & $9.56^{\mathrm{a}}$ & $9.33^{\mathrm{a}}$ & $8.78^{\mathrm{a}}$ \\
\hline & & Ambient & $9.22^{\mathrm{a}}$ & $11.44^{\mathrm{a}}$ & $10.78^{\mathrm{a}}$ & $9.44^{\mathrm{abc}}$ & - & - & - \\
\hline & \multirow{2}{*}{$\begin{array}{c}\text { Non- } \\
\text { perforated }\end{array}$} & Cold & $9.11^{\mathrm{a}}$ & $10.22^{\mathrm{b}-\mathrm{e}}$ & $9.89^{\mathrm{bc}}$ & $9.67^{\mathrm{ab}}$ & $9.33^{\mathrm{a}}$ & $9.00^{\mathrm{ab}}$ & $8.44^{\mathrm{ab}}$ \\
\hline & & Ambient & $9.22^{\mathrm{a}}$ & $10.11^{\mathrm{b}-\mathrm{f}}$ & $9.44^{\mathrm{b}-\mathrm{e}}$ & $8.89^{\text {def }}$ & $7.78^{\mathrm{c}}$ & - & - \\
\hline & \multirow{2}{*}{$\begin{array}{c}\text { Non- } \\
\text { packaged }\end{array}$} & Cold & $8.99^{\mathrm{a}}$ & $10.34^{\mathrm{bcd}}$ & $9.22^{\text {cde }}$ & - & - & - & - \\
\hline & & Ambient & $9.22^{\mathrm{a}}$ & $10.44^{\text {bcd }}$ & - & - & - & - & - \\
\hline \multirow{6}{*}{ Early Grande } & \multirow{2}{*}{ Perforated } & Cold & $9.11^{\mathrm{a}}$ & $10.56^{\mathrm{abc}}$ & $10.22^{\mathrm{ab}}$ & $9.89^{\mathrm{a}}$ & $9.44^{\mathrm{a}}$ & $8.56^{\mathrm{bc}}$ & $8.11^{\mathrm{bc}}$ \\
\hline & & Ambient & $9.11^{\mathrm{a}}$ & $10.89^{\mathrm{ab}}$ & $9.89^{\mathrm{bc}}$ & $8.89^{\mathrm{def}}$ & - & - & - \\
\hline & \multirow{2}{*}{$\begin{array}{c}\text { Non- } \\
\text { perforated }\end{array}$} & Cold & $9.11^{\mathrm{a}}$ & $9.33^{\mathrm{e}-\mathrm{h}}$ & $9.22^{\text {cde }}$ & $9.11^{\mathrm{cde}}$ & $8.78^{b}$ & $8.22^{\mathrm{cd}}$ & $7.89^{\mathrm{cd}}$ \\
\hline & & Ambient & $9.22^{\mathrm{a}}$ & $8.78^{\text {hij }}$ & $8.11^{\mathrm{fg}}$ & $7.89 \mathrm{~g}$ & $7.11^{\mathrm{d}}$ & - & - \\
\hline & \multirow{2}{*}{$\begin{array}{c}\text { Non- } \\
\text { packaged }\end{array}$} & Cold & $9.11^{\mathrm{a}}$ & $9.56^{\mathrm{d}-\mathrm{h}}$ & $9.11^{\text {cde }}$ & - & - & - & - \\
\hline & & Ambient & $9.22^{\mathrm{a}}$ & $8.22^{\mathrm{ijk}}$ & - & - & - & - & - \\
\hline \multicolumn{3}{|c|}{$\mathrm{SE} \pm$} & 0.34 & 0.33 & 0.28 & 0.19 & 0.17 & 0.17 & 0.16 \\
\hline \multicolumn{3}{|c|}{ CV (\%) } & 6.51 & 6.20 & 5.37 & 3.73 & 3.60 & 3.73 & 3.52 \\
\hline
\end{tabular}

Note: $\mathrm{N}: \mathrm{B}$ : $\mathrm{SE}=$ Standard error, $\mathrm{CV}=$ Coefficient of variation, means with different letters in a column indicate significant differences at $\mathrm{p}=0.05$.

\subsection{Fruit Firmness}

The interaction of packaging, storage and genotype had high significant $(\mathrm{p} \leq 0.001)$ effect on firmness of peach fruits during most of the storage time. Firmness of peach fruits varied between 1.17 in Early Grande in perforated plastic stored under ambient condition on day 20 to $4.67 \mathrm{~kg} \mathrm{~cm}^{-2}$ in cultivar $90-19 \mathrm{H}$ non-packaged and stored at ambient Table 7. In general, firmness decreased with storage time; but, variation among genotypes was observed under the different storage conditions. This might be due to activity of enzymes and degradation of pectic substances present in the fruits and loss of water. Galvis, et al. [62] and Drake, et al. [39] reported similar findings in pear fruits.

Table-7. Influence of plastic packaging and storage condition on firmness of peach genotypes during 30 days of storage.

\begin{tabular}{|c|c|c|c|c|c|c|c|c|c|}
\hline \multicolumn{3}{|c|}{ Treatments } & & \multicolumn{6}{|c|}{ Storage period(days) } \\
\hline Variety & $\begin{array}{c}\text { Plastic } \\
\text { Packaging }\end{array}$ & Storage & $\mathrm{O}$ & 5 & 10 & 15 & 20 & 25 & 30 \\
\hline \multirow[t]{6}{*}{ Tropic Beauty } & \multirow[t]{2}{*}{ Perforated } & Cold & $4.25^{\mathrm{a}}$ & $3.67^{\mathrm{c}}$ & $3.67^{\mathrm{fg}}$ & $3.33^{\mathrm{cd}}$ & $3.17^{\mathrm{b}}$ & $2.67^{\mathrm{a}}$ & $1.92^{\mathrm{bc}}$ \\
\hline & & Ambient & $4.5 \mathrm{O}^{\mathrm{a}}$ & $4.42^{\mathrm{ab}}$ & $3.50^{\mathrm{g}}$ & $3.12^{\mathrm{d}}$ & - & - & - \\
\hline & \multirow[t]{2}{*}{ Non-perforated } & Cold & $4.5 \mathrm{O}^{\mathrm{a}}$ & $4.42^{\mathrm{ab}}$ & $4.25^{\mathrm{abc}}$ & $4.00^{\mathrm{a}}$ & $3.58^{\mathrm{a}}$ & $2.5 \mathrm{O}^{\mathrm{ab}}$ & $1.67^{\mathrm{cd}}$ \\
\hline & & Ambient & $4.5 \mathrm{O}^{\mathrm{a}}$ & $4.67^{\mathrm{a}}$ & $4.25^{\mathrm{abc}}$ & $4.17^{\mathrm{a}}$ & $3.33^{\mathrm{ab}}$ & - & - \\
\hline & \multirow[t]{2}{*}{ Non-packaged } & Cold & $4.33^{\mathrm{a}}$ & $3.33^{\text {cde }}$ & $2.42^{\mathrm{j}}$ & - & - & - & - \\
\hline & & Ambient & $4.41^{\mathrm{a}}$ & $3.17^{\mathrm{ef}}$ & - & - & - & - & - \\
\hline \multirow[t]{6}{*}{$9 \mathrm{O}-19 \mathrm{H}$} & \multirow[t]{2}{*}{ Perforated } & Cold & $4.41^{\mathrm{a}}$ & $4.25^{\mathrm{b}}$ & $4.33^{\mathrm{ab}}$ & $3.67^{\mathrm{b}}$ & $3.17^{\mathrm{b}}$ & $2.25^{\mathrm{bc}}$ & $1.58^{\mathrm{cde}}$ \\
\hline & & Ambient & $4.5 \mathrm{O}^{\mathrm{a}}$ & $3.42^{\text {cde }}$ & $3.17^{\mathrm{h}}$ & $2.92^{\mathrm{e}}$ & - & - & - \\
\hline & \multirow[t]{2}{*}{ Non-perforated } & Cold & $4.5 \mathrm{O}^{\mathrm{a}}$ & $4.67^{\mathrm{a}}$ & $4.08^{\mathrm{bcd}}$ & $3.67^{\mathrm{b}}$ & $2.75^{\mathrm{cd}}$ & $2.00^{\mathrm{c}}$ & $1.42^{\mathrm{de}}$ \\
\hline & & Ambient & $4.41^{\mathrm{a}}$ & $4.67^{\mathrm{a}}$ & $3.92^{\text {def }}$ & $3.33^{\mathrm{cd}}$ & $2.75^{\mathrm{cd}}$ & - & - \\
\hline & \multirow[t]{2}{*}{ Non-packaged } & Cold & $4.41^{\mathrm{a}}$ & $3.58^{\mathrm{cd}}$ & $3.17^{\mathrm{h}}$ & - & - & - & - \\
\hline & & Ambient & $4.41^{\mathrm{a}}$ & $3.58^{\mathrm{cd}}$ & - & - & - & - & - \\
\hline \multirow[t]{6}{*}{ Bonnigold } & \multirow[t]{2}{*}{ Perforated } & Cold & $4.5 \mathrm{O}^{\mathrm{a}}$ & $4.58^{\mathrm{ab}}$ & $4.33^{\mathrm{ab}}$ & $3.50^{\mathrm{bc}}$ & $3.17^{\mathrm{b}}$ & $2.5 \mathrm{O}^{\mathrm{ab}}$ & $2.33^{\mathrm{a}}$ \\
\hline & & Ambient & $4.5 \mathrm{O}^{\mathrm{a}}$ & $3.25^{\mathrm{de}}$ & $3.08^{\mathrm{h}}$ & $2.83^{\mathrm{e}}$ & - & - & - \\
\hline & \multirow[t]{2}{*}{ Non-perforated } & Cold & $4.5 \mathrm{O}^{\mathrm{a}}$ & $4.5 \mathrm{O}^{\mathrm{ab}}$ & $4.00^{\text {cde }}$ & $3.50^{\mathrm{bc}}$ & $3.08^{\mathrm{bc}}$ & $2.58^{\mathrm{a}}$ & $2.17^{\mathrm{ab}}$ \\
\hline & & Ambient & $4.58^{\mathrm{a}}$ & $4.5 \mathrm{O}^{\mathrm{ab}}$ & $3.75^{\mathrm{efg}}$ & $3.42^{\mathrm{c}}$ & $2.67^{\mathrm{d}}$ & - & - \\
\hline & \multirow[t]{2}{*}{ Non-packaged } & Cold & $4.41^{\mathrm{a}}$ & $2.67^{\mathrm{gh}}$ & $1.33^{\mathrm{k}}$ & - & - & - & - \\
\hline & & Ambient & $4.41^{\mathrm{a}}$ & $3.08^{\text {ef }}$ & - & - & - & - & - \\
\hline \multirow[t]{6}{*}{ Early Grande } & \multirow[t]{2}{*}{ Perforated } & Cold & $4.5 \mathrm{O}^{\mathrm{a}}$ & $4.67^{\mathrm{a}}$ & $4.42^{\mathrm{a}}$ & $3.67^{\mathrm{b}}$ & $2.67^{\mathrm{d}}$ & $2.08^{\mathrm{c}}$ & $1.5 \mathrm{O}^{\mathrm{de}}$ \\
\hline & & Ambient & $4.5 \mathrm{O}^{\mathrm{a}}$ & $2.83^{\mathrm{fg}}$ & $2.50^{\mathrm{ij}}$ & $1.25^{\mathrm{g}}$ & - & - & - \\
\hline & \multirow[t]{2}{*}{ Non-perforated } & Cold & $4.41^{\mathrm{a}}$ & $4.42^{\mathrm{ab}}$ & $4.33^{\mathrm{ab}}$ & $3.42^{\mathrm{c}}$ & $2.67^{\mathrm{d}}$ & $2.17^{\mathrm{c}}$ & $1.25^{\mathrm{e}}$ \\
\hline & & Ambient & $4.41^{\mathrm{a}}$ & $4.5 \mathrm{O}^{\mathrm{ab}}$ & $2.75^{\mathrm{i}}$ & $1.92^{\mathrm{f}}$ & $1.17^{\mathrm{e}}$ & - & - \\
\hline & \multirow[t]{2}{*}{ Non-packaged } & Cold & $4.41^{\mathrm{a}}$ & $2.58^{\mathrm{gh}}$ & $1.42^{\mathrm{k}}$ & - & - & - & - \\
\hline & & Ambient & $4.58^{\mathrm{a}}$ & $2.33^{\mathrm{h}}$ & - & - & - & - & - \\
\hline \multicolumn{3}{|c|}{$\mathrm{SE} \pm$} & 0.16 & 0.15 & 0.09 & 0.08 & 0.12 & 0.11 & 0.11 \\
\hline \multicolumn{3}{|c|}{ CV (\%) } & 6.20 & 6.58 & 4.70 & 4.32 & 7.32 & 7.85 & 11.43 \\
\hline
\end{tabular}

Note: $\mathrm{SE}=$ Standard error, $\mathrm{CV}=$ Coefficient of variation, means with different letters in a column indicate significant differences at $\mathrm{p}=0.05$. 
The control recorded least firmness than the packed fruits in all varieties on days 5 and 10 . Until day 15 storage time, significant difference was observed in the different packaging materials; Tropic Beauty and 90-19H packaged with non-perforated bag were the most firm. Early Grande Fruits packaged with perforated as well as non-perforated plastic lost firmness sharply after 20 days while Tropic Beauty, Bonnigold and 90-19H kept in perforated and non-perforated bags remained firmer relatively longer. Under ambient storage environment varieties packed with non-perforated plastic were relatively firmer than perforated packed and the control.

In general, softening of fruits was experienced as the storage time advanced which might be due to texture modification due to degradation of polysaccharides that take place during ripening [42]. Texture changes in fruits are consequences of modifications by component polysaccharides that, in turn, give rise to disassembly of primary cell wall and middle lamella structures due to enzyme activity on carbohydrate polymers [63].

Therefore, the differences in decrease of firmness of peach fruits between treatments might be due to differences in rate of respiration that affect depolimerization of pectin's during ripening [64]. Perforated package kept firmness than non-perforated package and control in cold storage up to the end of storage period. However, the least fruit firmness in packages with non-perforated indicates high $\mathrm{CO}_{2}$ or low $\mathrm{O}_{2}$ injury that may result in fruit softening [65].The non-perforated package also retain firmness fruits than the control whereas at the ambient storage condition non-perforated packages maintain firmness better than perforated package and control at all days. Such influence of package may contribute to their retardation effects of ripening thorough reduction of oxygen concentration around the fruits as well as through reduction of water loss and keeping tissues more turgid [63].

Better firmness of fruits in cold storage could be due to the presence of higher relative humidity and lower temperature which in turn retard its rate of respiration and transpiration. Likewise, lower softening rate in packed fruits could be due to the packages in lowering its rate of respiration, ripening process and moisture loss [65]. The decrease in the fruit firmness with increment of storage could be due to hydrolysis of starch [66].

\subsection{Marketability (\%)}

Interaction of packages, storage conditions and genotype had highly significant $(\mathrm{p} \leq 0.001)$ effect on marketability of peach fruits Table 8 . The marketability (\%) of peach fruits remained $100 \%$ in all genotypes kept in non-perforated bags in the cold storage while fruit stored in perforated under cold storage were $100 \%$ marketable for up to day 25. Non packaged fruits kept under ambient condition had only 86-90\% marketability on day 5, which were discarded thereafter. However, perforated bags extended $100 \%$ fruit marketability up to day 10 under ambient storage while non-perforated bags extended by additional five days.

Under ambient condition and with no packaging, genotype 90-19H and Early Grande had more marketable fruits $(90 \%)$ than the other two which had $86.67 \%$ marketable fruits. Bonnigold fruits stored at ambient in nonperforated bags had 7-10 percent more marketability compared to the rest on day 20 of storage time. The shelf life termination of peaches stored at ambient condition was due to shriveling, over ripening, discoloration (decay) and mould growth. Shriveling and mould development were dominant in the control fruits and polyethylene packed fruits, respectively, with mould being more in perforated packages. Faster transpiration rate at higher temperature could result in shriveling of unpackaged fruits. Mainly, high respiration rate at high temperature can lead to senescence because of the stored food reserve exhaust [67].

Table-8. Influence of plastic package and storage condition on marketable percentage of peach genotypes during the storage.

\begin{tabular}{|c|c|c|c|c|c|c|c|c|c|}
\hline \multicolumn{3}{|c|}{ Treatments } & & \multicolumn{6}{|c|}{ Storage period (days) } \\
\hline$\overline{\text { Genotypes }}$ & $\begin{array}{c}\text { Plastic } \\
\text { Packaging }\end{array}$ & Storage & $\mathrm{O}$ & 5 & 10 & 15 & 20 & 25 & 30 \\
\hline \multirow[t]{6}{*}{ Tropic Beauty } & \multirow[t]{2}{*}{ Perforated } & Cold & $100.00^{\mathrm{a}}$ & $100.00^{\mathrm{a}}$ & $100.00^{\mathrm{a}}$ & $100.00^{\mathrm{a}}$ & $100.00^{\mathrm{a}}$ & $100.00^{\mathrm{a}}$ & $86.67^{\mathrm{c}}$ \\
\hline & & Ambient & $100.00^{\mathrm{a}}$ & $100.00^{\mathrm{a}}$ & $100.00^{\mathrm{a}}$ & $86.67^{\mathrm{b}}$ & - & - & - \\
\hline & \multirow[t]{2}{*}{ Non-perforated } & Cold & $100.00^{\mathrm{a}}$ & $100.00^{\mathrm{a}}$ & $100.00^{\mathrm{a}}$ & $100.00^{\mathrm{a}}$ & $100.00^{\mathrm{a}}$ & $100.00^{\mathrm{a}}$ & $100.00^{\mathrm{a}}$ \\
\hline & & Ambient & $100.00^{\mathrm{a}}$ & $100.00^{\mathrm{a}}$ & $100.00^{\mathrm{a}}$ & $100.00^{\mathrm{a}}$ & $76.67^{\mathrm{c}}$ & $13.33^{\mathrm{c}}$ & - \\
\hline & \multirow[t]{2}{*}{ Non-packaged } & Cold & $100.00^{\mathrm{a}}$ & $100.00^{\mathrm{a}}$ & $86.67^{\mathrm{bc}}$ & - & - & - & - \\
\hline & & Ambient & $100.00^{\mathrm{a}}$ & $86.67^{\mathrm{c}}$ & - & - & - & - & - \\
\hline \multirow[t]{6}{*}{$9 \mathrm{O}-19 \mathrm{H}$} & \multirow[t]{2}{*}{ Perforated } & Cold & $100.00^{\mathrm{a}}$ & $100.00^{\mathrm{a}}$ & $100.00^{\mathrm{a}}$ & $100.00^{\mathrm{a}}$ & $100.00^{\mathrm{a}}$ & $100.00^{\mathrm{a}}$ & $86.67^{\mathrm{c}}$ \\
\hline & & Ambient & $100.00^{\mathrm{a}}$ & $100.00^{\mathrm{a}}$ & $100.00^{\mathrm{a}}$ & $80.00^{\mathrm{c}}$ & - & - & - \\
\hline & \multirow[t]{2}{*}{ Non-perforated } & Cold & $100.00^{\mathrm{a}}$ & $100.00^{\mathrm{a}}$ & $100.00^{\mathrm{a}}$ & $100.00^{\mathrm{a}}$ & $100.00^{\mathrm{a}}$ & $100.00^{\mathrm{a}}$ & $100.00^{\mathrm{a}}$ \\
\hline & & Ambient & $100.00^{\mathrm{a}}$ & $100.00^{\mathrm{a}}$ & $100.00^{\mathrm{a}}$ & $100.00^{\mathrm{a}}$ & $76.67^{\mathrm{c}}$ & $10.00^{\mathrm{c}}$ & - \\
\hline & \multirow[t]{2}{*}{ Non-packaged } & Cold & $100.00^{\mathrm{a}}$ & $100.00^{\mathrm{a}}$ & $83.33^{\mathrm{c}}$ & - & - & - & - \\
\hline & & Ambient & $100.00^{\mathrm{a}}$ & $90.00^{\mathrm{b}}$ & - & - & - & - & - \\
\hline \multirow[t]{6}{*}{ Bonnigold } & \multirow[t]{2}{*}{ Perforated } & Cold & $100.00^{\mathrm{a}}$ & $100.00^{\mathrm{a}}$ & $100.00^{\mathrm{a}}$ & $100.00^{\mathrm{a}}$ & $100.00^{\mathrm{a}}$ & $100.00^{\mathrm{a}}$ & $93.33^{\mathrm{b}}$ \\
\hline & & Ambient & $100.00^{\mathrm{a}}$ & $100.00^{\mathrm{a}}$ & $100.00^{\mathrm{a}}$ & $90.00^{\mathrm{b}}$ & - & - & - \\
\hline & \multirow[t]{2}{*}{ Non-perforated } & Cold & $100.00^{\mathrm{a}}$ & $100.00^{\mathrm{a}}$ & $100.00^{\mathrm{a}}$ & $100.00^{\mathrm{a}}$ & $100.00^{\mathrm{a}}$ & $100.00^{\mathrm{a}}$ & $100.00^{\mathrm{a}}$ \\
\hline & & Ambient & $100.00^{\mathrm{a}}$ & $100.00^{\mathrm{a}}$ & $100.00^{\mathrm{a}}$ & $100.00^{\mathrm{a}}$ & $83.33^{\mathrm{b}}$ & $26.67^{\mathrm{b}}$ & - \\
\hline & \multirow[t]{2}{*}{ Non-packaged } & Cold & $100.00^{\mathrm{a}}$ & $100.00^{\mathrm{a}}$ & $90.00^{\mathrm{b}}$ & - & - & - & - \\
\hline & & Ambient & $100.00^{\mathrm{a}}$ & $86.67^{\mathrm{c}}$ & - & - & - & - & - \\
\hline \multirow[t]{6}{*}{ Early Grande } & \multirow[t]{2}{*}{ Perforated } & Cold & $100.00^{\mathrm{a}}$ & $100.00^{\mathrm{a}}$ & $100.00^{\mathrm{a}}$ & $100.00^{\mathrm{a}}$ & $100.00^{\mathrm{a}}$ & $100.00^{\mathrm{a}}$ & $90.00^{\mathrm{bc}}$ \\
\hline & & Ambient & $100.00^{\mathrm{a}}$ & $100.00^{\mathrm{a}}$ & $100.00^{\mathrm{a}}$ & $86.67^{\mathrm{b}}$ & - & - & - \\
\hline & \multirow[t]{2}{*}{ Non-perforated } & Cold & $100.00^{\mathrm{a}}$ & $100.00^{\mathrm{a}}$ & $100.00^{\mathrm{a}}$ & $100.00^{\mathrm{a}}$ & $100.00^{\mathrm{a}}$ & $100.00^{\mathrm{a}}$ & $100.00^{\mathrm{a}}$ \\
\hline & & Ambient & $100.00^{\mathrm{a}}$ & $100.00^{\mathrm{a}}$ & $100.00^{\mathrm{a}}$ & $100.00^{\mathrm{a}}$ & $73.33^{\mathrm{c}}$ & $13.33^{\mathrm{c}}$ & - \\
\hline & \multirow[t]{2}{*}{ Non-packaged } & Cold & $100.00^{\mathrm{a}}$ & $100.00^{\mathrm{a}}$ & $83.33^{\mathrm{c}}$ & - & - & - & - \\
\hline & & Ambient & $100.00^{\mathrm{a}}$ & $90.00^{\mathrm{b}}$ & - & - & - & - & - \\
\hline \multicolumn{3}{|c|}{$\mathrm{SE} \pm$} & - & 0.96 & 1.83 & 1.18 & 1.92 & 1.67 & 2.04 \\
\hline \multicolumn{3}{|c|}{$\mathrm{CV}(\%)$} & - & 1.70 & 3.25 & 2.12 & 3.60 & 4.0 & 3.74 \\
\hline
\end{tabular}

Note: $\mathrm{SE}=$ Standard error, $\mathrm{CV}=$ Coefficient of variation, means with different letters in a column indicate significant differences at $\mathrm{p}=0.05$. 
Peach fruits in cold storage remain fresh and firm for reasonable period of time. This could contribute to lower rate of respiration and transpiration due to relatively lower temperature and higher RH. Since high respiration decreases storage life [33] lower temperature storage is known to extending the storage life of perishable produce [68]. Generally, marketability of peaches stored in cold storage was better than those stored under ambient condition. Similar reports were stated in mango [34] and Aliye, et al. [43] and tomato [44].

Packed fruits had more marketability percentage than the control under both storage conditions while nonperforated packages had more percent marketable fruits than perforated packed ones. These effects could be due to modified atmosphere created inside the package as well as reduction in water loss [69]. Lower rates respiration and ethylene production, reduced action of ethylene, reduced ripening and senescence, slow growth of decay causing pathogens due to modification of gas atmosphere inside the package and low temperature that is not suit for microorganisms might be the reason to extend storage life of the fruits [70].

\subsection{Percent Decay}

The result for decay index is shown in Table 9. In non-packed fruits stored at ambient condition, the decay started after 5 days and reached maximum $(100 \%)$ on $20^{\text {th }}$ day of storage period. Perforated plastic packed fruits stored at ambient delayed total decay loss to 25 days. No decay was recorded in fruits packed with non-perforated plastic till 30 days of cold storage; this may be due to less chance of microorganism entry and lower rate of respiration that results in less moisture inside the packaging. Fruits in non-perforated bags had less decay than those in perforated ones. This lower decay might be due to limited permeability of gases $\left(\mathrm{CO}_{2}\right.$ and $\left.\mathrm{O}_{2}\right)$ and water vapour, which can interplay with physiological processes [29, 52]. The index also varied with the varieties where Bonnigold variety showed $7-15 \%$ more marketable fruits than the remaining ones in non-perforated bags stored at ambient after 25 days of storage. Packages can protect from contamination, damage and most importantly, against excess moisture loss; hence, maintained cell wall stability and middle lamella of the fruits [71]. Similar findings of plum fruits at low temperature were also reported [72].

\subsection{Specific Gravity}

The interaction effect between packaging materials, peach fruit genotypes and storage conditions had significant $(\mathrm{P} \leq 0.05)$ effect on the specific gravity Table 10. In this study, the $\mathrm{SG}$ value varied from 1.033 to 1.053. The highest specific gravity was recorded for non-perforated plastic packed and control (unpacked) treatments of Bonnigold variety under cold storage condition in perforated plastic bag on day 30 of storage time, whereas the lowest was for fruits of $90-19 \mathrm{H}$ with perforated plastic packed and stored in the cold storage and Tropic beauty with non-perforated and stored in ambient on day 5. However, fruits of Tropic Beauty under all treatments and 90-19H in perforated as well as non-perforated bags had statistically similar low value on $5^{\text {th }}$ day of storage.

In general, there was an increasing trend with storage time for each treatment, which is directly related with increase of total soluble solid of the fruits. There was highly significant $(p<0.001)$ difference between varieties throughout the storage period. At the end of the storage period Bonnigold (1.053), followed by Early Grande showed highest specific gravity of fruits packed and stored in cold storage. With increasing of oBrix reading there was positive relation of specific gravity; similar result was observed by Ting and Blair [73].

\subsection{Flesh Browning}

Cultivars showed very different flesh browning values Table 11 after day 20 of storage. Genotype 90-19H and Early Grande with non-perforated package had the highest values on day 25 and 30. Non-perforated plastic packed fruits were highly affected with flesh browning irrespective of variety except Bonnigold which did not show browning until the last storage time. This high browning of the fruits might be due to fruits high total phenolic content and polyphenol oxidase activities, since both of them are said to be the main factors responsible for enzymatic browning [74]. It has been shown that the rate of browning of fruit products depends on the nature, concentration and interaction of the phenolic compounds that are co-present in tissues [75]. Bonnigold didn't show any flesh color browning throughout the storage period of cold storage both on packed as well as nonpacked. A certain difference between varieties for these parameters was observed by some authors on peach [75] apple [76] and pear [77]. For all cultivars of perforated and non-perforated packed fruits, except Bonnigold variety, browning rose after day 20 of cold storage.

\section{Conclusions}

Non-perforated packed fruits had reduced weight loss compared to the control under both storage conditions. Towards the end of storage, non-perforated plastic packaged fruits in cold storage maintained more TSS, AA, TA, TSS/TA, pH and specific gravity content. Non-perforated bag with cold storage were generally more effective compared to perforated package and the control in maintaining the quality of the fruit. Except tropic beauty and Early Grande, non-perforated packaged fruits had higher TSS than perforated packed fruits at day 15 of ambient environment storage.

As the storage time progressed $\mathrm{pH}$ increased irrespective of treatment applied. TSS/TA increased throughout the storage period irrespective of treatments due to increased TSS and decreased TA values. Ascorbic acid content decreased as the storage time advanced irrespective of treatments in which cold storage maintains better than ambient condition. Overall, packaging combined with cold storage maintained the freshness, firmness and maintained the shelf life stability of peach fruits. 
Table-9. Influence of plastic package and storage condition on percent decay of peach genotypes during 30 days of storage

\begin{tabular}{|c|c|c|c|c|c|c|c|c|c|}
\hline \multicolumn{3}{|c|}{ Treatments } & & \multicolumn{6}{|c|}{ Storage period (days) } \\
\hline Genotypes & Plastic Packaging & Storage & 0 & 5 & 10 & 15 & 20 & 25 & 30 \\
\hline \multirow{6}{*}{$\begin{array}{l}\text { Tropic } \\
\text { Beauty }\end{array}$} & \multirow[t]{2}{*}{ Perforated } & Cold & 0.00 & 0.00 & 0.00 & 0.00 & 0.00 & 0.00 & $13.33\left(21.14^{\mathrm{b}}\right)$ \\
\hline & & Ambient & 0.00 & 0.00 & 0.00 & $13.33\left(21.14^{\mathrm{ef}}\right)$ & $76.67\left(61.22^{\mathrm{b}}\right)$ & $100.00\left(90.00^{\mathrm{a}}\right)$ & $100.00\left(90.00^{a}\right)$ \\
\hline & \multirow[t]{2}{*}{ Non-perforated } & Cold & 0.00 & 0.00 & 0.00 & 0.00 & 0.00 & 0.00 & 0.00 \\
\hline & & Ambient & 0.00 & 0.00 & 0.00 & 0.00 & $23.33\left(28.77^{\mathrm{ef}}\right)$ & $86.67\left(68.85^{\mathrm{b}}\right)$ & $100.00\left(90.00^{\mathrm{a}}\right)$ \\
\hline & \multirow[t]{2}{*}{ Non-packaged } & Cold & 0.00 & 0.00 & $13.33\left(21.14^{\mathrm{b}}\right)$ & $70.00\left(56.99^{\mathrm{c}}\right)$ & $100.00\left(90.00^{\mathrm{a}}\right)$ & $100.00\left(90.00^{\mathrm{a}}\right)$ & $100.00\left(90.00^{\mathrm{a}}\right)$ \\
\hline & & Ambient & 0.00 & $13.33\left(21.14^{\mathrm{a}}\right)$ & $80.00\left(63.93^{\mathrm{a}}\right)$ & $100.00\left(90.00^{\mathrm{a}}\right)$ & $100.00\left(90.00^{a}\right)$ & $100.00\left(90.00^{\mathrm{a}}\right)$ & $100.00\left(90.00^{\mathrm{a}}\right)$ \\
\hline \multirow[t]{6}{*}{$90-19 \mathrm{H}$} & \multirow[t]{2}{*}{ Perforated } & Cold & 0.00 & 0.00 & 0.00 & 0.00 & 0.00 & 0.00 & $13.33\left(21.14^{\mathrm{b}}\right)$ \\
\hline & & Ambient & 0.00 & 0.00 & 0.00 & $20.00\left(26.56^{\mathrm{e}}\right)$ & $80.00\left(63.93^{\mathrm{b}}\right)$ & $100.00\left(90.00^{\mathrm{a}}\right)$ & $100.00\left(90.00^{\mathrm{a}}\right)$ \\
\hline & \multirow[t]{2}{*}{ Non-perforated } & Cold & 0.00 & 0.00 & 0.00 & 0.00 & 0.00 & 0.00 & 0.00 \\
\hline & & Ambient & 0.00 & 0.00 & 0.00 & 0.00 & $23.33^{\text {ef }}$ & $90.00\left(71.56^{\mathrm{b}}\right)$ & $100.00\left(90.00^{\mathrm{a}}\right)$ \\
\hline & \multirow[t]{2}{*}{ Non-packaged } & Cold & 0.00 & 0.00 & $16.67\left(23.36^{\mathrm{b}}\right)$ & $80.00\left(63.93^{\mathrm{b}}\right)$ & $100.00\left(90.00^{\mathrm{a}}\right)$ & $100.00\left(90.00^{\mathrm{a}}\right)$ & $100.00\left(90.00^{\mathrm{a}}\right)$ \\
\hline & & Ambient & 0.00 & $10.00\left(18.43^{\mathrm{a}}\right)$ & $70.00\left(56.99^{\mathrm{a}}\right)$ & $100.00\left(90.00^{\mathrm{a}}\right)$ & $100.00\left(90.00^{a}\right)$ & $100.00\left(90.00^{\mathrm{a}}\right)$ & $100.00\left(90.00^{a}\right)$ \\
\hline \multirow[t]{6}{*}{ Bonnigold } & \multirow[t]{2}{*}{ Perforated } & Cold & 0.00 & 0.00 & 0.00 & 0.00 & 0.00 & 0.00 & $6.67\left(12.29^{\mathrm{c}}\right)$ \\
\hline & & Ambient & 0.00 & 0.00 & 0.00 & $10.00\left(18.43^{\mathrm{f}}\right)$ & $56.67\left(48.85^{\mathrm{d}}\right)$ & $100.00\left(90.00^{\mathrm{a}}\right)$ & $100.00\left(90.00^{\mathrm{a}}\right)$ \\
\hline & \multirow[t]{2}{*}{ Non-perforated } & Cold & 0.00 & 0.00 & 0.00 & 0.00 & 0.00 & 0.00 & 0.00 \\
\hline & & Ambient & 0.00 & 0.00 & 0.00 & 0.00 & $16.67\left(23.85^{\mathrm{f}}\right)$ & $73.33\left(59.00^{\mathrm{c}}\right)$ & $100.00\left(90.00^{\mathrm{a}}\right)$ \\
\hline & \multirow[t]{2}{*}{ Non-packaged } & Cold & 0.00 & 0.00 & $10.00\left(18.43^{b}\right)$ & $53.33\left(46.92^{\mathrm{d}}\right)$ & $100.00\left(90.00^{a}\right)$ & $100.00\left(90.00^{\mathrm{a}}\right)$ & $100.00\left(90.00^{a}\right)$ \\
\hline & & Ambient & 0.00 & $13.33\left(12.29^{\mathrm{a}}\right)$ & $70.00\left(53.07^{\mathrm{a}}\right)$ & $100.00\left(90.00^{\mathrm{a}}\right)$ & $100.00\left(90.00^{\mathrm{a}}\right)$ & $100.00\left(90.00^{\mathrm{a}}\right)$ & $100.00\left(90.00^{a}\right)$ \\
\hline \multirow{6}{*}{$\begin{array}{c}\text { Early } \\
\text { Grande }\end{array}$} & \multirow[t]{2}{*}{ Perforated } & Cold & 0.00 & 0.00 & 0.00 & 0.00 & 0.00 & 0.00 & $10.00\left(18.43^{\mathrm{b}}\right)$ \\
\hline & & Ambient & 0.00 & 0.00 & 0.00 & $13.33\left(21.14^{\mathrm{ef}}\right)$ & $66.67\left(54.78^{\mathrm{c}}\right)$ & $100.00\left(90.00^{\mathrm{a}}\right)$ & $100.00\left(90.00^{a}\right)$ \\
\hline & \multirow[t]{2}{*}{ Non-perforated } & Cold & 0.00 & 0.00 & 0.00 & 0.00 & 0.00 & 0.00 & 0.00 \\
\hline & & Ambient & 0.00 & 0.00 & 0.00 & 0.00 & $26.67\left(30.99^{\mathrm{e}}\right)$ & $86.67\left(68.85^{\mathrm{b}}\right)$ & $100.00\left(90.00^{\mathrm{a}}\right)$ \\
\hline & \multirow[t]{2}{*}{ Non-packaged } & Cold & 0.00 & 0.00 & $16.67\left(23.85^{\mathrm{b}}\right)$ & $73.33\left(59.00^{\mathrm{bc}}\right)$ & $100.00\left(90.00^{\mathrm{a}}\right)$ & $100.00\left(90.00^{\mathrm{a}}\right)$ & $100.00\left(90.00^{a}\right)$ \\
\hline & & Ambient & 0.00 & $10.00\left(18.43^{\mathrm{a}}\right)$ & $73.33\left(59.00^{\mathrm{a}}\right)$ & $100.00\left(90.00^{\mathrm{a}}\right)$ & $100.00\left(90.00^{a}\right)$ & $100.00\left(90.00^{\mathrm{a}}\right)$ & $100.00\left(90.00^{\mathrm{a}}\right)$ \\
\hline \multicolumn{3}{|c|}{$\mathrm{SE} \pm$} & - & 3.36 & 3.62 & 2.14 & 1.82 & 1.11 & 1.62 \\
\hline \multicolumn{3}{|c|}{$\mathrm{CV}(\%)$} & - & 33.09 & 15.70 & 6.59 & 4.75 & 2.27 & 3.71 \\
\hline
\end{tabular}


Table-10. Influence of plastic packaging and storage condition on specific gravity of peach genotypes during 30 days of storage.

\begin{tabular}{|c|c|c|c|c|c|c|c|c|c|}
\hline \multicolumn{3}{|c|}{ Treatments } & & \multicolumn{6}{|c|}{ Storage period(days) } \\
\hline Variety & $\begin{array}{c}\text { Plastic } \\
\text { Packaging }\end{array}$ & Storage & $\bar{O}$ & 5 & 10 & 15 & 20 & 25 & 30 \\
\hline \multirow[t]{6}{*}{ Tropic Beauty } & \multirow[t]{2}{*}{ Perforated } & Cold & $1.034^{\mathrm{a}}$ & $1.036^{\mathrm{f}-\mathrm{i}}$ & $1.037 \mathrm{gh}$ & $1.040 \mathrm{gh}$ & $1.043^{\mathrm{de}}$ & $1.044^{\mathrm{d}}$ & $1.045^{\mathrm{d}}$ \\
\hline & & Ambient & $1.033^{\mathrm{a}}$ & $1.035^{\mathrm{ghi}}$ & $1.036^{\mathrm{h}}$ & $1.041 \mathrm{gh}$ & - & - & - \\
\hline & \multirow{2}{*}{$\begin{array}{c}\text { Non- } \\
\text { perforated }\end{array}$} & Cold & $1.033^{\mathrm{a}}$ & $1.034^{\mathrm{hi}}$ & $1.035^{\mathrm{h}}$ & $1.039^{\mathrm{h}}$ & $1.041^{\mathrm{e}}$ & $1.042^{\mathrm{e}}$ & $1.043^{\mathrm{e}}$ \\
\hline & & Ambient & $1.034^{\mathrm{a}}$ & $1.033^{\mathrm{i}}$ & $1.038^{\mathrm{gh}}$ & $1.039^{\mathrm{h}}$ & $1.042^{\mathrm{e}}$ & - & - \\
\hline & \multirow[t]{2}{*}{ Non-packaged } & Cold & $1.034^{\mathrm{a}}$ & $1.037^{\mathrm{e}-\mathrm{i}}$ & $1.038^{\mathrm{gh}}$ & - & - & - & - \\
\hline & & Ambient & $1.035^{\mathrm{a}}$ & $1.037^{\mathrm{e}-\mathrm{i}}$ & - & - & - & - & - \\
\hline \multirow[t]{6}{*}{$9 \mathrm{O}-19 \mathrm{H}$} & \multirow[t]{2}{*}{ Perforated } & Cold & $1.034^{\mathrm{a}}$ & $1.033^{\mathrm{i}}$ & $1.037^{\mathrm{gh}}$ & $1.041^{\mathrm{gh}}$ & $1.043^{\mathrm{de}}$ & $1.043^{\mathrm{de}}$ & $1.044^{\mathrm{de}}$ \\
\hline & & Ambient & $1.033^{\mathrm{a}}$ & $1.035^{\mathrm{ghi}}$ & $1.04 \mathrm{O}^{\mathrm{fg}}$ & $1.041 \mathrm{gh}$ & - & - & - \\
\hline & \multirow{2}{*}{$\begin{array}{c}\text { Non- } \\
\text { perforated }\end{array}$} & Cold & $1.034^{\mathrm{a}}$ & $1.035^{\mathrm{ghi}}$ & $1.037 \mathrm{gh}$ & $1.040 \mathrm{gh}$ & $1.043^{\mathrm{de}}$ & $1.043^{\mathrm{de}}$ & $1.044^{\mathrm{de}}$ \\
\hline & & Ambient & $1.035^{\mathrm{a}}$ & $1.036^{\mathrm{f}-\mathrm{i}}$ & $1.037^{\mathrm{gh}}$ & $1.042^{\mathrm{fg}}$ & $1.043^{\mathrm{de}}$ & - & - \\
\hline & \multirow[t]{2}{*}{ Non-packaged } & Cold & $1.034^{\mathrm{a}}$ & $1.040^{\text {cde }}$ & $1.042^{\mathrm{ef}}$ & - & - & - & - \\
\hline & & Ambient & $1.035^{\mathrm{a}}$ & $1.037^{\mathrm{e}-\mathrm{i}}$ & - & - & - & - & - \\
\hline \multirow[t]{6}{*}{ Bonnigold } & \multirow[t]{2}{*}{ Perforated } & Cold & $1.035^{\mathrm{a}}$ & $1.039^{\mathrm{c}-\mathrm{f}}$ & $1.045^{\mathrm{b}-\mathrm{e}}$ & $1.051^{\mathrm{a}}$ & $1.051^{\mathrm{a}}$ & $1.052^{\mathrm{a}}$ & $1.053^{\mathrm{a}}$ \\
\hline & & Ambient & $1.034^{\mathrm{a}}$ & $1.042^{\mathrm{bc}}$ & $1.045^{\mathrm{b}-\mathrm{e}}$ & $1.046^{\text {cde }}$ & - & - & - \\
\hline & \multirow{2}{*}{$\begin{array}{c}\text { Non- } \\
\text { perforated }\end{array}$} & Cold & $1.034^{\mathrm{a}}$ & $1.041^{\mathrm{cd}}$ & $1.043^{\mathrm{c}-\mathrm{f}}$ & $1.049^{\mathrm{ab}}$ & $1.049^{\mathrm{abc}}$ & $1.051^{\mathrm{b}}$ & $1.051^{\mathrm{b}}$ \\
\hline & & Ambient & $1.035^{\mathrm{a}}$ & $1.038^{\mathrm{d}-\mathrm{g}}$ & $1.046^{\mathrm{bc}}$ & $1.048^{\mathrm{bcd}}$ & $1.047^{\mathrm{bc}}$ & - & - \\
\hline & \multirow[t]{2}{*}{ Non-packaged } & Cold & $1.034^{\mathrm{a}}$ & $1.045^{\mathrm{ab}}$ & $1.053^{\mathrm{a}}$ & - & - & - & - \\
\hline & & Ambient & $1.034^{\mathrm{a}}$ & $1.045^{\mathrm{ab}}$ & - & - & - & - & - \\
\hline \multirow[t]{6}{*}{ Early Grande } & \multirow[t]{2}{*}{ Perforated } & Cold & $1.033^{\mathrm{a}}$ & $1.043^{\mathrm{bc}}$ & $1.046^{\mathrm{bcd}}$ & $1.048^{\mathrm{bcd}}$ & $1.05 \mathrm{O}^{\mathrm{ab}}$ & $1.050^{\mathrm{b}}$ & $1.051^{\mathrm{b}}$ \\
\hline & & Ambient & $1.036^{\mathrm{a}}$ & $1.047^{\mathrm{a}}$ & $1.048^{\mathrm{b}}$ & $1.050^{\mathrm{ab}}$ & - & - & - \\
\hline & \multirow{2}{*}{$\begin{array}{c}\text { Non- } \\
\text { perforated }\end{array}$} & Cold & $1.034^{\mathrm{a}}$ & $1.041^{\mathrm{cd}}$ & $1.043^{\mathrm{def}}$ & $1.045^{\mathrm{ef}}$ & $1.046^{\mathrm{cd}}$ & $1.046^{\mathrm{c}}$ & $1.048^{\mathrm{c}}$ \\
\hline & & Ambient & $1.034^{\mathrm{a}}$ & $1.038^{\mathrm{d}-\mathrm{g}}$ & $1.043^{\mathrm{c}-\mathrm{f}}$ & $1.045^{\mathrm{ef}}$ & $1.047^{\mathrm{bc}}$ & - & - \\
\hline & \multirow[t]{2}{*}{ Non-packaged } & Cold & $1.034^{\mathrm{a}}$ & $1.049^{\mathrm{a}}$ & $1.051^{\mathrm{a}}$ & - & - & - & - \\
\hline & & Ambient & $1.034^{\mathrm{a}}$ & $1.042^{\mathrm{bc}}$ & - & - & - & - & - \\
\hline \multicolumn{3}{|c|}{$\mathrm{SE} \pm$} & 0.034 & 0.001 & 0.001 & 0.001 & 0.001 & 0.001 & 0.0004 \\
\hline \multicolumn{3}{|c|}{ CV (\%) } & 0.18 & 0.22 & 0.18 & 0.16 & 0.17 & 0.08 & 0.08 \\
\hline
\end{tabular}

Note: $\mathrm{SE}=$ Standard error, $\mathrm{CV}=$ Coefficient of variation, different letters in a column indicate significant differences at $\mathrm{P}=0.05$.

Table-11. Influence of plastic packaging and storage condition on flesh color browning of peach genotypes during 30 days of storage

\begin{tabular}{|c|c|c|c|c|c|c|c|c|c|}
\hline \multicolumn{3}{|c|}{ Treatments } & & \multicolumn{6}{|c|}{ Storage period (days) } \\
\hline Genotypes & Plastic Packaging & Storage & $\mathrm{O}$ & 5 & 10 & 15 & 20 & 25 & 30 \\
\hline \multirow{6}{*}{ Tropic Beauty } & \multirow[t]{2}{*}{ Perforated } & Cold & 1.00 & 1.00 & 1.00 & 1.00 & 1.00 & $1.17^{\mathrm{bcd}}$ & $1.58^{\mathrm{d}}$ \\
\hline & & Ambient & 1.00 & 1.00 & 1.00 & 1.00 & - & - & - \\
\hline & \multirow[t]{2}{*}{ Non-perforated } & Cold & 1.00 & 1.00 & 1.00 & 1.00 & 1.00 & $1.25^{\mathrm{bc}}$ & $2.08^{\mathrm{b}}$ \\
\hline & & Ambient & 1.00 & 1.00 & 1.00 & 1.00 & 1.00 & - & - \\
\hline & \multirow[t]{2}{*}{ Non-packaged } & Cold & 1.00 & 1.00 & 1.00 & - & - & - & - \\
\hline & & Ambient & 1.00 & 1.00 & - & - & - & - & - \\
\hline \multirow{6}{*}{$90-19 \mathrm{H}$} & \multirow[t]{2}{*}{ Perforated } & Cold & 1.00 & 1.00 & 1.00 & 1.00 & 1.00 & $1.08^{\mathrm{cd}}$ & $1.33^{\mathrm{e}}$ \\
\hline & & Ambient & 1.00 & 1.00 & 1.00 & 1.00 & - & - & - \\
\hline & \multirow[t]{2}{*}{ Non-perforated } & Cold & 1.00 & 1.00 & 1.00 & 1.00 & 1.00 & $1.33^{\mathrm{ab}}$ & $1.83^{\mathrm{c}}$ \\
\hline & & Ambient & 1.00 & 1.00 & 1.00 & 1.00 & 1.00 & - & - \\
\hline & \multirow[t]{2}{*}{ Non-packaged } & Cold & 1.00 & 1.00 & 1.00 & - & - & - & - \\
\hline & & Ambient & 1.00 & 1.00 & - & - & - & - & - \\
\hline \multirow{6}{*}{ Bonnigold } & \multirow[t]{2}{*}{ Perforated } & Cold & 1.00 & 1.00 & 1.00 & 1.00 & 1.00 & $1.00^{\mathrm{d}}$ & $1.00^{f}$ \\
\hline & & Ambient & 1.00 & 1.00 & 1.00 & 1.00 & - & - & - \\
\hline & \multirow[t]{2}{*}{ Non-perforated } & Cold & 1.00 & 1.00 & 1.00 & 1.00 & 1.00 & $1.00^{\mathrm{d}}$ & $1.00^{\mathrm{f}}$ \\
\hline & & Ambient & 1.00 & 1.00 & 1.00 & 1.00 & 1.00 & - & - \\
\hline & \multirow[t]{2}{*}{ Non-packaged } & Cold & 1.00 & 1.00 & 1.00 & - & - & - & - \\
\hline & & Ambient & 1.00 & 1.00 & - & - & - & - & - \\
\hline \multirow{6}{*}{ Early Grande } & \multirow[t]{2}{*}{ Perforated } & Cold & 1.00 & 1.00 & 1.00 & 1.00 & 1.00 & $1.33^{\mathrm{ab}}$ & $1.75^{\mathrm{cd}}$ \\
\hline & & Ambient & 1.00 & 1.00 & 1.00 & 1.00 & - & - & - \\
\hline & \multirow[t]{2}{*}{ Non-perforated } & Cold & 1.00 & 1.00 & 1.00 & 1.00 & 1.00 & $1.5 \mathrm{O}^{\mathrm{a}}$ & $2.58^{\mathrm{a}}$ \\
\hline & & Ambient & 1.00 & 1.00 & 1.00 & 1.00 & 1.00 & - & - \\
\hline & \multirow[t]{2}{*}{ Non-packaged } & Cold & 1.00 & 1.00 & 1.00 & - & - & - & - \\
\hline & & Ambient & 1.00 & 1.00 & - & - & - & - & - \\
\hline \multicolumn{3}{|c|}{$\mathrm{SE} \pm$} & - & - & - & - & - & 0.08 & 0.07 \\
\hline \multicolumn{3}{|c|}{$\mathrm{CV}(\%)$} & - & - & - & - & - & 11.17 & 6.93 \\
\hline
\end{tabular}

Note: $\mathrm{SE}=$ Standard error, $\mathrm{CV}=$ Coefficient of variation, means with different letters in a column indicate significant differences at $\mathrm{p}=0.05$

The percentage marketability of peach fruits remained $100 \%$ in all genotypes kept in non-perforated bags in the cold storage while fruit stored in perforated under cold storage were $100 \%$ marketable for up to day 25 . All genotypes with non-perforated packaging under cold storage did not show any sign of decay while perforated packaging exposed fruits to decay. In non-packaged fruits stored under ambient condition severity of decay started from day five onwards reaching $100 \%$ starting from day 15 . Flesh color browning was not experienced only in Bonnigold in perforated and non-perforated packaging under cold store.

Based on the result of the present study, peach genotypes had different response to packaging materials and storage environment. Over all, use of non-perforated plastic packaging and cold store extend shelf life by maintaining the quality of peach during post-harvest storage compared to perforated packaging and control. 


\section{References}

[1] J. La Rue, Introduction, In: La Rue, J.H. and Johnson, R.S. (Eds) Peaches, Plums, and Nectarines: Growing and handling for fresh market. Publication No. 3331. Oakland, California: University of California, Division of Agriculture and Natural Resources, 1989.

D. Byrne, "Peach breeding trends," Acta Hort, vol. 592, pp. 49-59, 2002.

[3] C. M. Cantin, M. A. Moreno, and Y. Gogorcena, "Evaluation of the antioxidant capacity, phenolic compounds, and vitamin C content of different peach and nectarine ‘Prunus persica (L.) Batsch־ breeding progenies," Journal of Agricultural and Food Chemistry, vol. 57, pp. 4586-4592, 2009. Available at: https://doi.org/10.1021/jf900385a.

[4] D. Ashebir, T. Deckers, J. Nyssen, W. Bihon, A. Tsegay, H. Tekie, J. Poesen, M. Haile, F. Wondumagegneheu, and D. Raes, "Growing apple (Malus domestica) under tropical mountain climate conditions in Northern Ethiopia," Experimental Agriculture, vol. 46, pp. 53-65, 2010. Available at: https://doi.org/10.1017/s0014479709990470.

[5] FAOSTAT (Food and Agricultural Organization), "Fact fish peaches and nectarines production quantity for Ethiopia," Nerwsletter, 2016.

[6] EARO (Ethiopian Agricultural Research Organization), Food science and postharvest technology research strategy. Nazareth, Ethiopia: EARO, 2000

[7] E. Kebede, "Processing of horticultural produces in Ethiopia," Acta Hort, vol. 270, pp. 270-301, 1991. Available at: https://doi.org/10.17660/actahortic.1991.270.36

[8] G. Tonini and E. Tura, "Influence of storage and shelf-life time on rots of peaches and nectarines," in International Postharvest Science Conference Postharvest, 1996, pp. 520-520.

[9] R. E. Hardenburg, A. E. Watada, and C. Yang, "The commercial storage of fruits, vegetables, and florist and nursery stocks," Agriculture Handbook (Washington), vol. 66, pp. 12-62, 1990.

[10] WFLO (World Food Logistics Organization), "Commodity storage manual peaches," Neweletter, 2008.

[11] K. R. Day, "Orchard factors affecting postharvest stone fruit quality," Hort Science, vol. 32, pp. 820-823, 1997. Available at: https://doi.org/10.21273/hortsci.32.5.820

[12] S. Lurie and C. H. Crisosto, "Chilling injury in peach and nectarine," Postharvest Biology and Technology, vol. 37, pp. 195-208, 2005. Available at: https://doi.org/10.1016/j.postharvbio.2005.04.012.

[13] C. L. Girardi, A. R. Corrent, L. Lucchetta, M. R. Zanuzo, T. S. da Costa, A. Brackmann, R. M. Twyman, F. R. Nora, L. Nora, and J. A. Silva, "Effect of ethylene, intermittent warming and controlled atmosphere on postharvest quality and the occurrence of woolliness in peach (Prunus persica cv. Chiripá) during cold storage," Postharvest Biology and Technology, vol. 38, pp. 25-33, 2005. Available at: https://doi.org/10.1016/j.postharvbio.2005.05.007.

[14] L. Kitinoja, "Returnable plastic crate (RPC) systems can reduce postharvest losses and improve earnings for fresh produce operations," The Postharvest Education Foundation (PEF), White Paper No. 13-O1, 2013.

[15] M. Erkan and C. Y. Wang, "Modified and controlled atmosphere storage of subtropical crops," Stewart Postharvest Review, vol. 5, pp. 1-8, 2006. Available at: https://doi.org/10.2212/spr.2006.5.4.

[16] L. Kitinoja, S. Saran, S. K. Roy, and A. A. Kader, "Postharvest technology for developing countries: Challenges and opportunities in research, outreach and advocacy," Journal of the Science of Food and Agriculture, vol. 91, pp. 597-603, 2011. Available at: https://doi.org/10.1002/jsfa.4295.

[17] J. Lammertyn, N. Scheerlinck, P. Jancsók, B. Verlinden, and B. Nicolar, "A respiration-diffusion model for 'Conference'pears I: Model development and validation," Postharvest Biology and Technology, vol. 30, pp. 29-42, 2003. Available at: https://doi.org/10.1016/s0925-5214(03)00061-9.

[18] EIAR (Ethiopian Institute of Agricultural Research), Holeta agricultural research center progress report. Holeta, Ethiopia: EIAR, 2017.

[19] A. Kader, "Fruit maturity, ripening and quality relationships," Acta Hort, vol. 485, pp. 203-208, 1999. Available at: https://doi.org/10.17660/ActaHortic.1999.485.27.

[20] M. Mohammed, L. Wilson, and P. Gomes, "Postharvest sensory and physiochemical attributes of processing and nonprocessing tomato cultivars," Journal of Food Quality, vol. 22, pp. 167-182, 1999. Available at: https://doi.org/10.1111/j.17454557.1999.tbo0549.x.

[21] D. Garner, C. Crisosto, P. Wiley, and G. Crisosto, Measurement of pH and titratable acidity. Quality evaluation methodology. USA: Kearney Agricultural Center, 2005.

[22] O. A. Bessey and C. King, "The distribution of vitamin C in plant and animal tissues, and its determination," Journal of Biological Chemistry, vol. 103, pp. 687-698, 1933.

[23] A. Kader, L. Morris, and P. Chen, "Evaluation of two objective methods and a subjective rating scale for measuring tomato fruit firmness," Journal of the American Society for Horticultural Science, vol. 103, pp. 70-73, 1978

[24] Y. Jiang, D. C. Joyce, and A. J. Macnish, "Extension of the shelf life of banana fruit by 1-methylcyclopropene in combination with polyethylene bags," Postharvest Biology and Technology, vol. 16, pp. 187-193, 1999. Available at: https://doi.org/10.1016/s0925$5214(99) 00009-5$.

[25] K. Colin, "Refractometers," Newsletter, 2017

[26] T. J. Frett, K. Gasic, J. R. Clark, D. Byrne, T. Gradziel, and C. Crisosto, "Standardized phenotyping for fruit quality in peach (Prunus persica (L.) Batsch)," Journal of the American Pomological Society, vol. 66, p. 214, 2012.

[27] A. Kader, "Biochemical and physiological basis for effects of controlled atmospheres on fruits and vegetables," Food Technol, vol. 40, pp. 99-104, 1986.

[28] G. Calvo, M. Salvador, and E. Sanchez, "Control of superficial scald in _Beurred'Anjou' pears with low oxygen levels," Acta Hort, vol. 596, pp. 879-882, 2002. Available at: https://doi.org/10.17660/actahortic.2002.596.153.

[29] L. Tijskens and H. Vollebregt, "Passive and semi-active modified atmosphere packaging of prickly pear cactus stems (Opuntis spp.)," Acta Hort, vol. 604, pp. 665-668, 2003.

[30] P. Lam, "Respiration rate, ethylene production and skin color change of papaya at different temperatures," Acta Hort, vol. 269, pp. 257-266, 1990. Available at: https://doi.org/10.17660/actahortic.1990.269.33.

[31] R. Pal, S. Roy, and S. Srivastava, "Storage performance of kinnow mandarins in evaporative cool chamber and ambient condition," Journal of Food Science and Technology, vol. 34, pp. 200-203, 1997.

[32] E. Proulx, M. Cecilia, N. Nunes, J. Emond, and J. K. Brecht, "Quality attributes limiting papaya postharvest life at chilling and nonchilling temperatures," in Proceedings of the Florida State Horticultural Society. Florida State Horticultural Society, 2005, pp. $389-395$.

[33] L. Lee, J. Arul, R. Lencki, and F. Castaigne, "A review on modified atmosphere packaging and preservation of fresh fruits and vegetables: Physiological basis and practical aspects-Part I," Packaging Technology and Science, vol. 8, pp. 315-331, 1995. Available at: https://doi.org/10.1002/pts.2770080605.

[34] Tilahunsiyum and W. Kebede, "Forced ventilation evaporative cooling: A case study on banana, papaya, orange, mandarin, and lemon," Journal of Tropical Agriculture, vol. 81, pp. 1-6, 2004.

[35] A. Nath, B. C. Deka, A. Singh, R. Patel, D. Paul, L. Misra, and H. Ojha, "Extension of shelf life of pear fruits using different packaging materials," Journal of Food Science and Technology, vol. 49, pp. 556-563, 2012. Available at: https://doi.org/10.1007/s13197-011-0305-4.

[36] F. Mathooko, "A comparison of modified atmosphere packaging under ambient conditions and low temperatures storage on quality of tomato fruit," African Journal of Food, Agriculture, Nutrition and Development, vol. 3, pp. 20-27, 2003.

[37] S. Ben-Yehoshua, "Individual seed packaging of fruits and vegetables in plastic film: New postharvest technique," The Journal of Horticultural Science, vol. 20, pp. 32-37, 1985.

[38] C. Ribeiro, P. Nazare, J. Sobreiro, and R. Veltman, "Influence of orchard, harvest date and controlled atmosphere, on storage quality of -Rochallpear," Acta Hort, vol. 599, pp. 639-645, 2003. Available at: https://doi.org/10.17660/actahortic.2003.599.83.

[39] S. Drake, E. Mielke, and D. Elfving, "Maturity and storage quality of concorde pears," Hort Technology, vol. 14, pp. 250-256, 2004.

[40] C. Crisosto and A. Kader, "Scientific name and introduction," University of California Davis, CA 95616, 1999. 
[41] L. Carrillo, H. A. Cruz, L. F. Guevara, and L. O. Paredes, "Physico-chemical changes during ripening in storage of two varieties of prickly pear stored at $18^{\circ} \mathrm{C}, "$ Journal of Food Science and Technology, vol. 40, pp. 461-464, 2003.

[42] S. Irtwange, "Application of modified atmosphere packaging and related technology in postharvest handling of fresh fruits and vegetables," International Journal of Agricultural and Engineering, vol. 4, pp. 1-12, 2006.

[43] T. Aliye, T. Seyoum, and K. Woldetsadik, "Effect of disinfection, packaging, and storage environment on the shelf life of mango," Biosystems Engineering, vol. 96, pp. 201-212, 2007. Available at: https://doi.org/10.1016/j.biosystemseng.2006.10.006.

[44] G. Hirut, T. Seyoum, and K. Woldetsadik, "The effect of cultivar, maturity stage and storage environment on quality of tomatoes," Journal of Food Engineering, vol. 87, pp. 467-478, 2008. Available at: https://doi.org/10.1016/j.jfoodeng.2007.12.031.

[45] B. Akbudak and A. Eris, "Physical and chemical changes in peaches and nectarines during the modified atmosphere storage," Food Control, vol. 15, pp. 307-313, 2004. Available at: https://doi.org/10.1016/s0956-7135(03)00082-3.

[46] I. Agar, L. Son, and N. Kaska, "Postharvest physiology of some nectarine cultivars," Journal of the Faculty of Agriculture, Cukurova University, vol. 9, pp. 1-16, 1994.

[47] T. Fernandez and F. Artes, "Intermittent warming during cold storage of peaches packed in perforated polypropylene," LWT-Food Science and Technology, vol. 31, pp. 38-43, 1998.

[48] R. Wills, W. Mcglasson, D. Graham, H. Tlee, and E. Hall, Postharvest-An introduction to the physiology and handling of fruit and vegetables, 3rd ed. vol. 270. New York: VanNostrand Reinhold, 1989.

[49] Y. Selvaraj, D. Pal, M. Subramanyam, and C. Iyer, "Changes in the chemical composition of four cultivars of papaya (Carica papaya L.) during growth and development," Journal of Horticultural Science, vol. 57, pp. 135-143, 1982. Available at: https://doi.org/10.1080/00221589.1982.11515033.

[50] P. Agozzino, G. Avellone, F. Filizzola, V. Farina, and R. L. Bianco, "Changes in quality parameters and volatile aroma compounds in fairtime' peach during fruit development and ripening," Italian Journal of Food Science, vol. 19, pp. 3-13, 2007.

[51] A. Pongener, B. Mahajan, and H. Singh, "Effect of different packaging films on storage life and quality of peach fruits under cold storage conditions," Indian Journal of Horticulture, vol. 68, pp. 240-245, 2011.

[52] R. C. Soliva-Fortuny and O. Martín-Belloso, "Microbiological and biochemical changes in minimally processed fresh-cut Conference pears," European Food Research and Technology, vol. 217, pp. 4-9, 2003. Available at: https://doi.org/10.1007/s00217003-0701-8.

[53] P. Zerbini, "The quality of pear fruit," Acta Hort, vol. 596, pp. 805-810, 2002.

[54] A. Kader, Postharvest biology and technology: An overview. In Postharvest Technology of Horticultural Crops. In: Kader, A.A, Kasmire, R.F. Mitchell, F.G., Reid, M.S., Sommer, W.F. and Thompson, J.F. (Eds) Special Publication. 3311. Davis, CA: University of California, 1985.

[55] I. U. Bron and A. P. Jacomino, "Ripening and quality of 'golden'papaya fruit harvested at different maturity stages," Brazilian Journal of Plant Physiology, vol. 18, pp. 389-396, 2006. Available at: https://doi.org/10.1590/s 1677-04202006000300005.

[56] K. Mkhathini, L. Magwaza, T. Workneh, and G. Mwithiga, "Determinations of physical and chemical properties of Kwa ZuluNatal's household white peach 'landrace'in relation to extension services-a case study of Impendle local municipality," South African Journal of Agricultural Extension, vol. 45, pp. 95-109, 2017. Available at: https://doi.org/10.17159/2413-3221/2017/v45n 1a439.

[57] M. Petriccione, F. Mastrobuoni, M. Pasquariello, L. Zampella, E. Nobis, G. Capriolo, and M. Scortichini, "Effect of chitosan coating on the postharvest quality and antioxidant enzyme system response of strawberry fruit during cold storage," Foods, vol. 4, pp. 501523, 2015. Available at: https://doi.org/10.3390/foods4040501.

[58] A. Zanella, "Control of apple superficial scald and ripening-a comparison between 1-methylcyclopropene and diphenylamine postharvest treatments, initial low oxygen stress and ultra low oxygen storage," Postharvest Biology and Technology, vol. 27, pp. 6978, 2003. Available at: https://doi.org/10.1016/s0925-5214(02)00187-4.

[59] D. H. Byrne, A. N. Nikolic, and E. E. Burns, "Variability in sugars, acids, firmness, and color characteristics of 12 peach genotypes," Journal of the American Society for Horticultural Science, vol. 116, pp. 1004-1006, 1991. Available at: https://doi.org/10.21273/jashs.1 16.6.1004.

[60] B. Mahajan, W. Dhillon, K. Mahesh, and S. Bikramjit, "Effect of different packaging films on shelf life and quality of peach under super and ordinary market conditions," Journal of Food Science and Technology, vol. 52, pp. 3756-3762, 2015.

[61] O. Yoshida, H. Nakagawa, N. Ogura, and T. Sato, "Effect of heat treatment on the development of polygalacturonase activity in tomato fruit during ripening," Plant and Cell Physiology, vol. 25, pp. 505-509, 1984. Available at: https://doi.org/10.1093/oxfordjournals.pcp.a076739.

[62] S. Galvis, AC, S. Fonseca, A. Morais, and F. X. Malcata, "Physicochemical and sensory evaluation of 'Rocha'pear following controlled atmosphere storage," Journal of Food Science, vol. 68, pp. 318-327, 2003. Available at: https://doi.org/10.1111/j.13652621.2003.tb 14159.x.

[63] G. D. Manrique and F. M. Lajolo, "Cell-wall polysaccharide modifications during postharvest ripening of papaya fruit (Carica papaya)," Postharvest Biology and Technology, vol. 33, pp. 11-26, 2004. Available at: https://doi.org/10.1016/j.postharvbio.2004.01.007.

[64] H. Lazan, M. K. Selamat, and Z. M. Ali, " $\beta$-Galactosidase, polygalacturonase and pectinesterase in differential softening and cell wall modification during papaya fruit ripening," Physiologia Plantarum, vol. 95, pp. 106-112, 1995. Available at: https://doi.org/10.1034/j.1399-3054.1995.950116.x.

[65] D. Zagory and A. A. Kader, "Modified atmosphere packaging of fresh produce," Food Technol, vol. 42, pp. 70-77, 1988.

[66] A. Mattoo, T. Murata, E. Pantastico, K. Chachiss, K. Ogata, and C. Phan, Chemical changes during ripening and senescence In: Pantastico, E.B. (Ed) Post-harvest physiology, handling and utilization of tropical and subtropical fruits and vegetables. Westport Connecticut: The AVI Pub. Co. Inc, 1975.

[67] R. Paull, Pineaple and papaya.In: Symour, G.B., Taylor, J.E.and Tucker, G.A. (Eds) Biochemistry of fruit ripening. New York: Chapman and Hall, 1993.

[68] A. Exama, J. Arul, R. Lencki, and Z. Li, "Suitability of various plastic films for modified atmosphere packaging of fruits and vegetables: Gas transfer properties and effect of temperature fluctuation," Physiological Basis of Postharvest Technologies, vol. 343, pp. 175-180, 1992. Available at: https://doi.org/10.17660/actahortic.1993.343.39.

[69] G. Gonzalez-Aguilar, J. G. Buta, and C. Wang, "Methyl jasmonate and modified atmosphere packaging (MAP) reduce decay and maintain postharvest quality of papaya 'sunrise'," Postharvest Biology and Technology, vol. 28, pp. 361-370, 2003. Available at: https://doi.org/10.1016/s0925-52 14(02)00200-4.

[70] A. Kader and R. Rolle, "The role of postharvest management in assuring the quality and safety of horticultural produce," $F A O$ Agriculture Sup Systems Div, vol. 152, pp. 1010-1365, 2004.

[71] P. Hernandez-Munoz, E. Almenar, V. Del Valle, D. Velez, and R. Gavara, "Effect of chitosan coating combined with postharvest calcium treatment on strawberry (Fragaria $\times$ ananassa) quality during refrigerated storage," Food Chemistry, vol. 110, pp. 428-435, 2008. Available at: https://doi.org/10.1016/j.foodchem.2008.02.020.

[72] G. Manganaris, A. Vicente, and C. Crisosto, "Effect of pre-harvest and post-harvest conditions and treatments on plum fruit quality," CAB Reviews: Perspectives in Agriculture, Veterinary Science, Nutrition and Natural Resources, vol. 3, pp. 1-9, 2008. Available at: https://doi.org/10.1079/pavsnnr20083009.

[73] S. Ting and J. Blair, "The relation of specific gravity of whole fruit to the internal quality of qranges," Florida Agriculture Exp. Stations Journal Series No. 2217 , 1965.

[74] Q. He and Y. Luo, "Enzymatic browning and its control in fresh-cut produce," Stewart Postharvest Review, vol. 3, pp. 1-7, 2007. Available at: https://doi.org/10.2212/spr.2007.6:16.

[75] C. Y. Lee, V. Kagan, A. W. Jaworski, and S. K. Brown, "Enzymic browning in relation to phenolic compounds and polyphenoloxidase activity among various peach cultivars," Journal of Agricultural and Food Chemistry, vol. 38, pp. 99-101, 1990. Available at: https://doi.org/10.1021/jfooo91a019.

[76] M. Murata, M. Tsurutani, M. Tomita, S. Homma, and K. Kaneko, "Relationship between apple ripening and browning: Changes in polyphenol content and polyphenol oxidase," Journal of Agricultural and Food Chemistry, vol. 43, pp. 1115-1121, 1995. Available at: https://doi.org/10.1021/jfo0053a001. 
[77] E. Arias, J. González, P. López-Buesa, and R. Oria, "Optimization of processing of fresh-cut pear," Journal of the Science of Food and Agriculture, vol. 88, pp. 1755-1763, 2008. Available at: https://doi.org/10.1002/jsfa.3276. 